\title{
GEOGRAPHICAL DIVERSIFICATION AND LONGEVITY RISK MITIGATION IN ANNUITY PORTFOLIOS
}

\author{
BY
}

\author{
Clemente De Rosa, Elisa luciano and LuCA Regis (iD
}

\begin{abstract}
This paper provides a method to assess the risk relief deriving from a foreign expansion by a life insurance company. We build a parsimonious continuoustime model for longevity risk that captures the dependence across different ages in domestic versus foreign populations. We calibrate the model to portray the case of a UK annuity portfolio expanding internationally toward Italian policyholders. The longevity risk diversification benefits of an international expansion are sizable, in particular when interest rates are low. The benefits are judged based on traditional measures, such as the Risk Margin or volatility reduction, and on a novel measure, the Diversification Index.
\end{abstract}

\section{KEYWORDS}

Geographical diversification, life insurance, risk management, multipopulation mortality, longevity risk modeling, Solvency II capital requirements.

JEL codes: F23, F65, G15, G22.

*The authors thank the Global Risk Institute (Toronto) for financial support and its workshop participants in January 2017 for helpful suggestions. They thank participants and discussants in the 9th Financial Risk International Forum (Paris, March 2016), the 15th International Conference on Pensions, Insurance and Savings (Paris, May 2017), the Workshop on "Recent Developments in Dependence Modelling with Applications in Finance and Insurance" (Aegina, September 2018) as well as participants to the University of Florence inaugural Master lecture in October 2017, and the University of Verona day in honor of F. Rossi, for useful discussions and remarks. Financial support from the Italian Ministry of Education, University and Research (MIUR), "Dipartimenti di Eccellenza" grant 2018-2022 is gratefully acknowledged.

Astin Bulletin 51(2), 375-410. doi:10.1017/asb.2021.12 (c) 2021 by Astin Bulletin. All rights reserved. This is an Open Access article, distributed under the terms of the Creative Commons Attribution licence (http://creativecommons.org/licenses/by/4.0/), which permits unrestricted re-use, distribution, and reproduction in any medium, provided the original work is properly cited. 


\section{INTRODUCTION}

In the last 20 years, insurance companies have been expanding internationally, via subsidiaries operating in different countries or via cross-border mergers and acquisitions. From the middle of the Nineties, in Europe, due to the creation of a common regulatory framework, and in the US as well, cross-border expansions and $\mathrm{M} \& \mathrm{~A}$ operations in the insurance sector trended upward (see Ma and Pope, 2003). Cummins et al. (1999) argue that geographical diversification was a primary determinant of mergers and acquisitions in the US insurance industry in the nineties because geographically diversified firms were more likely to be the target of acquisitions. The largest insurers and reinsurers are indeed multinational companies, with subsidiaries and branches located in several countries. Schoenmaker and Sass (2016) report that in Europe, as of 2012, the share of cross-border activity in the insurance sector is higher than in the banking one and that the degree of internationalization of the 25 largest European insurers increased over the period 2000-2012, despite the financial crisis. The OECD, as of 2014, reports that on average around 30\% of the gross premiums of life insurance companies in 20 European countries, United States, and Japan comes from foreign-based controlled undertakings. Reinsurance companies have traditionally been more geographically diversified than insurance companies (Cummins and Xie, 2008) because their portfolios are more easily disconnected from the geographical localization of their branches.

An interesting, but, up to our knowledge, so far overlooked effect of internationalization for life insurers and reinsurers is longevity risk pooling. Indeed, even if - in expectation - longevity has been steadily increasing on a worldwide scale, idiosyncratic longevity risks of different populations are different and may be nonperfectly correlated across countries. As a result, pooling portfolios of policies written on the lives of different populations allows to diversify longevity risk.

The present paper aims at filling a gap in the literature by showing that the standard, solvency-based measures of riskiness can be lowered, especially in low-interest-rate environment, thanks to longevity diversification, and that even non-VaR-based risk decreases. To this end, we first introduce a novel parsimonious model for the joint mortality dynamics of policyholders in different countries.

We measure the benefits of longevity risk pooling for specific insurance companies using two standard measures, namely the change in the SolvencyII risk margin and in the volatility of the mortality intensity. We introduce and compute a novel diversification index, which can be defined thanks to our longevity model, because it stems from the correlation structure of different populations and cohorts within them. The three measures, analyzed together, provide complementary views to assess the risk effects of the international expansion. While the risk margin reduction, which was analyzed by De Rosa 
et al. (2018), offers a measure of the mitigation of the "tail" risk because it represents the loss in a worst-case scenario occurring with a low probability, the diversification index provides a synthetic indicator of how dissimilar from the initial portfolio the portfolio obtained after the expansion is.

In a numerical application, which portrays the situation of a UK annuity provider that can expand to Italy, we first assess that the model is able to fit well the observed mortality rates of individuals aged 65-75 years in the two populations, while capturing, using the Gaussian mapping technique, the imperfect correlations observed across ages and populations. Based on our model estimates, we then compute our international diversification measures for different portfolio expansions. We show that the risk margin can be as high as $3 \%$ as a proportion of the actuarial value, in the case of a foreign expansion, targeted to those cohorts in the Italian population who have low covariance with the initial annuity portfolio. We also highlight that longevity risk mitigation effects are more sizable when the interest rate - a flat term structure, for simplicity is lower.

The paper unfolds as follows. Section 2 reviews the background literature on multipopulation longevity modeling. Section 3 describes the set-up and the problem of the insurer. Section 4 presents our longevity risk model. Section 5 defines the longevity risk diversification measures and their application to assess the benefits of geographical diversification. Section 6 provides a calibrated application, computes the diversification measures for various portfolio choices, and provides sensitivity analysis to relevant parameters. Appendix A details the Gaussian mapping technique used to estimate the correlation structure, while Appendix B compares two ways of achieving the international diversification: a physical one, in which a foreign affiliate is opened, and a synthetic one, through a longevity swap.

\section{BACKGROUND LONGEVITY LITERATURE}

To model the risk of longevity, that is, the risk that policyholders live longer than expected, we set ourselves in the well-established continuous-time stochastic mortality setting initiated by Milevsky and Promislow (2001) that models the death of individuals as a Cox process. The time to death of an individual belonging to cohort $x_{i}$ is the first jump time of a Poisson process with stochastic intensity.

The literature on multipopulation models is rich in the discrete-time setting (for an overview, see Enchev et al., 2017). The well-known Lee and Carter (1992) model has been extended to describe the joint mortality of multiple populations. The seminal paper by Li and Lee (2005) introduced the modeling of different populations within the Lee-Carter model by assuming "coherence", that is, convergence in the long run, due to a common driving factor for every population. In their model, the correlation structure is based on a common period effect across populations, leading to perfect correlation among their 
ages (see Haberman et al., 2014). More recently, Yang and Wang (2013) proposed a different specification of the Li and Lee (2005) model, which accounts for population-specific components and a richer correlation structure:

$$
\log \left(\mu_{t, x}^{(i)}\right)=\alpha_{x}^{(i)}+B_{x}^{(i)} \cdot K_{t}^{(i)}+\epsilon_{x, t}^{(i)},
$$

where $\alpha_{x}^{(i)}, B_{x}^{(i)}, \epsilon_{x, t}^{(i)} \in \mathbb{R}$ are the idiosyncratic component, common factor, and error term, respectively. All the components are population-specific, and the errors $\epsilon_{x, t}^{i}$ are correlated across populations and ages. The number of parameters to be estimated obviously increases: two parameters per each age of each population, plus the parameters of the multivariate stochastic processes $K_{t}^{(i)}$ for $i=1, \ldots N$, plus a (symmetric) $N \times N$ matrix of parameters to describe the error terms for each age (i.e. $(N \cdot(N-1)) / 2) \times M$ parameters, where $M$ is the number of ages considered. However, it provides increasing modeling flexibility.

Fewer multipopulation models so far have taken a continuous-time approach, following the insights of Dahl et al. (2008). Nonetheless, continuoustime models, which provide a natural stochastic extension to the traditional actuarial mortality laws, have proved to be flexible and accurate in fitting the mortality surface of single populations (see, for instance, Luciano et al., 2008). The main advantage of continuous-time longevity modeling, especially when the mortality intensity is an affine process, is the possibility of having survival probabilities, both single and joint, in closed form, as a function of the longevity intensity and dependence parameters. This gives the possibility to compute in the same form hedging ratios for longevity risk hedging (see for instance Luciano et al., 2012). Last but not least, the price of survival bonds and longevity derivatives obtains quite easily. Another advantage is the possibility of coupling the affine longevity intensity with an affine modeling of instantaneous interest rate, which is the most renowned form of interest-rate modeling in Finance. As a result, closed-form expressions for reserves and fair values obtain, together with joint hedges of interest rate and longevity risk (see again Luciano et al., 2012). Affine interest-rate modeling gained popularity in the Financial Community, both Academic and non, because of its analytical properties, useful for valuation, optimization (hedging), and calibration. In the same vein, affine survival modeling seems a promising modeling avenue.

The model we develop below extends the basis-risk model presented in De Rosa et al. (2017) to a multipopulation setting. Both the basis-risk model and the one we are going to develop below are a stochastic extension of the deterministic Gompertz mortality law, a benchmark in the classical modeling of mortality arrival rates. Jevtić and Regis (2019) and Sherris et al. (2020) use stochastic processes belonging to the affine class to model the mortality of multiple cohorts and populations. While these papers propose applications based on the use of three Brownian risk sources, we assume as many dependent risk factors as domestic generations and an idiosyncratic source that drives the mortality intensity of the foreign population. Thus, we are able to capture 
the correlation structure of different generations within and across populations accurately, similarly to Yang and Wang (2013) in discrete time while preserving a good level of parsimony, especially when the number of populations considered increases. In our model, also, the mortality intensities of single cohorts and generations follow square-root processes, and therefore cannot become negative in our setting, while they are Gaussian in Sherris et al. (2020). This desirable feature implied by non-gaussianity, combined with our rich dependence structure, limits the availability of analytical formulas for covariances across ages and populations. Indeed, by allowing for an approximation proposed in the credit risk domain by Brigo and Alfonsi (2005) and widely adopted, we are able to provide closed-form expressions for variances and covariances within and across populations.

\section{SET-UP}

We consider a filtered probability space $(\Omega, \mathcal{F}, \mathbb{P})$, endowed with the usual properties, where $\mathcal{F}$ is the filtration containing the information regarding all the relevant variables and $\mathbb{P}$ is the historical probability measure. In this probability space, the mortality intensities of individuals are described as stochastic processes, and longevity risk, that is the risk of unexpected fluctuations in the likelihood of deaths of individuals, arises. In what follows, we will consider longevity risk as the only source of risk in our setup.

We consider an Annuity Provider, or Life-Insurer, based in a certain country (that we call domestic), having a portfolio of deferred annuities written on different cohorts belonging to the domestic population. Let $\mathcal{X}=\left\{x_{1}, \ldots, x_{m}\right\}$ be the set of annuitants' ages at time zero, and let $n_{i}$, for $i=1, \ldots, m$, be the number of annuities sold to people aged $x_{i}$. When an annuity is sold at time zero, the annuitant pays the initial premium. We compute the actuarial value of the liabilities net of that premium. After signing the contract, the annuitant will receive a series of fixed annual instalments $R$, starting from the year-end of his 65th birthday if $x_{i}<65$, or immediately if $x_{i} \geq 65$, until his death, that may happen at most when he reaches a final age $\omega$.

\subsection{Portfolio value}

Consistently with the Solvency II regulation, we write the overall value $\Pi^{0}(t)$ of the liability portfolio of a life insurer at time $t$ is the sum of two components: the Actuarial Value $A V_{\Pi^{0}}(t)$, which is the sum of the actuarial values of each individual contract $N_{i}(t)$ and represents a best estimate of the liabilities of the insurer, and the Risk Margin $R M_{\Pi^{0}}(t)$ of the portfolio itself:

$$
\Pi^{0}(t)=A V_{\Pi^{0}}(t)+R M_{\Pi^{0}}(t)=\sum_{i=1}^{m} n_{i} N_{i}(t)+R M_{\Pi^{0}}(t) .
$$


The actuarial value of the contract is its fair premium. Let $\tau=\max (65-$ $x_{i}, 0$ ) be the number of years before the individual $i$ aged $x_{i}$ reaches age 65 . If $\tau>0$, then the contract is a deferred annuity, while if $\tau=0$ the contract is an immediate annuity. Because we consider no risk source other then longevity risk, the actuarial value of an annuity can be expressed as

$$
N_{i}(t)=D(t, k) S_{i}(t, k)\left[R \sum_{u=1}^{\omega-k} D(k, k+u) S_{i}(k, k+u)\right],
$$

where $k=t+\tau, D(t, s), s \geq t$ denotes the deterministic financial discount factor, $D(t, s)=e^{-r(s-t)}, r \in \mathbb{R}$ and $S_{i}(t, \cdot)$ is the time- $t$ survival probability curve of the individual aged $x_{i}$ at time $t$.

We define the portfolio risk margin $R M_{\Pi^{0}}(t)$ as the discounted Value-atRisk, at a given confidence level $\alpha \in(0,1)$, of the unexpected portfolio's future actuarial value at a given time horizon $T$ :

$$
\begin{aligned}
R M_{\Pi^{0}}(t)= & D(t, t+T) \cdot \operatorname{VaR}_{\alpha}\left(A V_{\Pi^{0}}(t+T)-\mathbb{E}_{t}\left[A V_{\Pi^{0}}(t+T)\right]\right), \\
= & D(t, t+T) \cdot \inf \left\{l \in \mathbb{R}^{+}: \mathbb{P}\left(A V_{\Pi^{0}}(t+T)\right.\right. \\
& \left.\left.-\mathbb{E}_{t}\left[A V_{\Pi^{0}}(t+T)\right]>l\right)<1-\alpha\right\},
\end{aligned}
$$

where $\mathbb{P}(\cdot)$ denotes the probability of the event that the future actuarial value exceeds its time-t expected value by more than $l$.

\subsection{Portfolio expansion}

We consider the case in which the Insurer wants to expand the size of her annuity portfolio and can choose between two alternative strategies. The first one consists simply in selling new contracts to her own domestic population. In this case, we denote with $n_{i}^{\prime}$ the number of new contracts sold to individuals aged $x_{i}$, with $\Pi^{D}$ the portfolio composed of just these new annuities, and with $\Pi^{1}$ the portfolio after the expansion, composed of the old and the new contracts. The actuarial value of the new portfolio is simply

$$
A V_{\Pi^{D}}(t)=\sum_{i=1}^{m} n_{i}^{\prime} N_{i}(t)
$$

and

$$
A V_{\Pi^{1}}(t)=A V_{\Pi^{0}}(t)+A V_{\Pi^{D}}(t) .
$$

The value of the total portfolio $\Pi^{1}$ is the sum of the actuarial value of the old portfolio, the actuarial value of the new portfolio and the risk margin of the total portfolio:

$$
\Pi^{1}(t)=A V_{\Pi^{1}}(t)+R M_{\Pi^{1}}(t)=A V_{\Pi^{0}}(t)+A V_{\Pi^{D}}(t)+R M_{\Pi^{1}}(t) .
$$


The second possible strategy is to acquire a new portfolio of annuities $\Pi^{F}$, written on a foreign population. We assume that, for each age $x_{i}$, the number of annuities written on people aged $x_{i}$ in the foreign population is $n_{i}^{f}$. The actuarial value of portfolio $\Pi^{F}$ is

$$
A V_{\Pi^{F}}(t)=\sum_{i=1}^{m} n_{i}^{f} N_{i}^{F}(t) .
$$

We denote with $\Pi^{2}$ the portfolio obtained after the expansion toward the foreign country. The actuarial value of such portfolio is

$$
A V_{\Pi^{2}}(t)=A V_{\Pi^{0}}(t)+A V_{\Pi^{F}}(t)
$$

and its overall value is

$$
\Pi^{2}(t)=A V_{\Pi^{2}}(t)+R M_{\Pi^{2}}(t)=A V_{\Pi^{0}}(t)+A V_{\Pi^{F}}(t)+R M_{\Pi^{2}}(t) .
$$

Notice that the original portfolio and the one obtained after the expansion do not have the same actuarial value, neither when the expansion is domestic nor foreign. The risk margin of the two portfolios is different as well.

\section{LONGEVITY RISK MODELING}

In this section, we propose a novel, parsimonious continuous-time model to describe the evolution of the mortality intensities of several cohorts in two different populations. The intensity of one population (the "foreign" one) is a linear combination of the other population's intensity (the benchmark, "domestic" one) and of an idiosyncratic risk factor. This makes the whole correlation structure across populations dependent on the weight of the linear combination.

To preserve tractability, we adopt stochastic processes belonging to the affine family. To ensure non-negativity of the intensities, we consider squareroot processes of the Cox et al. (1985) type for the intensities. In defining the features of the model, we aim at:

- accounting for different generations and populations parsimoniously;

- being able to compute the correlations between mortality intensities of different populations ${ }^{1}$;

- preserving as far as possible analytical tractability, while guaranteeing mortality intensity nonnegativity;

- being able to define a measure of overall (not tail) longevity diversification of different populations explicitly.

The model will reach these aims. In De Rosa et al. (2017), it is shown that its single-population version can be coupled with one of the best known models for interest rate risk (the CIR model) and still give analytic solutions, so 
that it could be extended to compute the VaR from longevity and interest rate risk. It also permits the computation of sensitivities and hedging ratios (greeks) explicitly. In our more complex multipopulation version, we are still able to obtain closed-form expressions for average survival probabilities and variances. However, the use of non-Gaussian processes limits the availability of closed-form expressions for the whole Variance/Covariance structure. To reconstruct them, we use an approximation technique applied by Brigo and Alfonsi (2005) in the credit risk domain.

\subsection{Mortality intensities and survival probabilities}

Let us consider two populations, each containing $m$ different cohorts. The first population is called the domestic population and the second one is called the foreign population. A given cohort $i$, with $i=1, \ldots, m$, belonging to one of the two populations, is identified by the (common) initial age $x_{i}$ at time zero. The set $\mathcal{X}$ of initial ages is common to the two populations.

\section{Domestic population}

The mortality intensity of each cohort $x_{i}$, for $i=1, \ldots, m$, belonging to the domestic population is denoted with $\lambda_{i}^{d}$, and follows a nonmean reverting CIR process:

$$
d \lambda_{i}^{d}(t)=\left(a_{i}+b_{i} \lambda_{i}^{d}(t)\right) d t+\sigma_{i} \sqrt{\lambda_{i}^{d}(t)} d W_{i}(t),
$$

where $a_{i}, b_{i}, \sigma_{i}, \lambda_{i}^{d}(0) \in \mathbb{R}^{++}$are strictly positive real constants and the $W_{i}$ 's are instantaneously correlated standard Brownian Motions: $d W_{i}(t) d W_{j}(t)=\rho_{i j} d t$ with $i, j \in\{1, \ldots, m\}$. As a consequence, the mortality intensities of two different cohorts belonging to the domestic population are instantaneously correlated, as soon as $\rho_{i, j} \neq 0$.

\section{Foreign population}

The mortality intensity of cohort $x_{i}$ belonging to the foreign population is denoted with $\lambda_{i}^{f}$ and is given by the convex combination of the mortality intensity of the corresponding cohort belonging to the domestic population $\lambda_{i}^{d}$ and an idiosyncratic component $\lambda^{\prime}$, which affects the foreign population only and that depends on the initial age $x_{i}$ in a deterministic way, ${ }^{2}$ that is,

$$
\lambda_{i}^{f}(t)=\delta_{i} \lambda_{i}^{d}(t)+\left(1-\delta_{i}\right) \lambda^{\prime}\left(t ; x_{i}\right)
$$

where

$$
d \lambda^{\prime}\left(t ; x_{i}\right)=\left(a^{\prime}+b^{\prime} d \lambda^{\prime}\left(t ; x_{i}\right)\right) d t+\sigma^{\prime} \sqrt{d \lambda^{\prime}\left(t ; x_{i}\right)} d W^{\prime}(t),
$$

with $\delta_{i} \in[0,1] .^{3}$ The functions $a^{\prime}, b^{\prime}$ and $\sigma^{\prime}$ are positive constants, while $W^{\prime}$ is a standard Brownian Motion, that is assumed to be independent of $W_{i}$ for each $i=1, \ldots, N$. 
Intuitively, the idiosyncratic risk source $W^{\prime}$ is population-specific, in the sense that it is common to all the cohorts of the foreign population. Nonetheless, each foreign cohort $x_{i}$ has a specific sensitivity to the idiosyncratic component $\lambda^{\prime}\left(t ; x_{i}\right)$ that is given by the parameter $\delta_{i}$, which is, instead, cohort-specific. The mortality intensities of two different cohorts of the foreign population are correlated, and the correlation between $\lambda_{i}^{f}$ and $\lambda_{j}^{f}$ depends both on the correlation between $\lambda_{i}^{d}$ and $\lambda_{j}^{d}$ and on the weights $\delta_{i}$ and $\delta_{j}$. Moreover, thanks to the presence of the idiosyncratic component $\lambda^{\prime}$ affecting the foreign population, our model allows to account for the nonperfect correlation between cohorts across the two populations. The correlation structure among the different cohorts of the two populations will be derived in Appendix A.

From (4.1) we have that the survival probability of generation $x_{i}$ in the domestic population is given by

$$
S_{i}^{d}(t, T)=A_{i}^{d}(t, T) e^{-B_{i}^{d}(t, T) \lambda_{i}^{d}(t)},
$$

where

$$
\begin{aligned}
& A_{i}^{d}(t, T)=\left(\frac{2 \gamma_{i} e^{\frac{1}{2}\left(\gamma_{i}-b_{i}\right)(T-t)}}{\left(\gamma_{i}-b_{i}\right)\left(e^{\gamma_{i}(T-t)}-1\right)+2 \gamma_{i}}\right)^{\frac{2 a_{i}}{\sigma_{i}^{2}}}, \\
& B_{i}^{d}(t, T)=\frac{2\left(e^{\gamma_{i}(T-t)}-1\right)}{\left(\gamma_{i}-b_{i}\right)\left(e^{\gamma_{i}(T-t)}-1\right)+2 \gamma_{i}},
\end{aligned}
$$

with $\gamma_{i}=\sqrt{b_{i}^{2}+2 \sigma_{i}^{2}}$. Similarly, for the foreign population we have

$$
S_{i}^{f}(t, T)=A_{i}^{d}(t, T) A^{\prime}(t, T) e^{-B_{i}^{d}(t, T) \delta_{i} \lambda_{i}^{d}(t)-B^{\prime}(t, T)\left(1-\delta_{i}\right) \lambda_{i}^{\prime}(t)},
$$

where

$$
\begin{aligned}
& A^{\prime}(t, T)=\left(\frac{2 \gamma^{\prime} e^{\frac{1}{2}\left(\gamma^{\prime}-b^{\prime}\right)(T-t)}}{\left(\gamma^{\prime}-b^{\prime}\right)\left(e^{\gamma^{\prime}(T-t)}-1\right)+2 \gamma^{\prime}}\right)^{\frac{2 a^{\prime}}{\left.\sigma^{\prime}\right)^{2}}}, \\
& B^{\prime}(t, T)=\frac{2\left(e^{\gamma^{\prime}(T-t)}-1\right)}{\left(\gamma^{\prime}-b^{\prime}\right)\left(e^{\gamma^{\prime}(T-t)}-1\right)+2 \gamma^{\prime}},
\end{aligned}
$$

with $\gamma^{\prime}=\sqrt{\left(b^{\prime}\right)^{2}+2\left(\sigma^{\prime}\right)^{2}}$.

Appendix A studies the Variance/Covariance Structure of the domestic intensities, the foreign ones, and the covariance of the domestic and foreign. It adopts an approximation, introduced and widely adopted in the credit risk domain (Brigo and Mercurio, 2001), known as Guassian mapping (see Brigo and Alfonsi, 2005), which also provides us with clear advantages in the calibration procedure. 


\section{MEASURING THE LONGEVITY RISK EFFECTS OF GEOGRAPHICAL DIVERSIFICATION}

In the following paragraphs, we comment on some measures of longevity risk in a portfolio, which allow us to appreciate the degree of geographical diversification achieved through a foreign expansion of the annuity portfolio. The first measure is the Percentage Risk Margin of the portfolio, computed à la Solvency II. Comparing this measure before and after a portfolio expansion allows to appreciate the economic benefit of a foreign expansion. A reduction in the percentage risk margin is connected with a reduction of tail risk, evaluated as the portfolio loss in a worst-case scenario.

The second measure we comment on is the Standard Deviation of the Portfolio Mortality Intensity, which is the intensity as a weighted average of the cohort-based mortality intensities entering the portfolio. A reduction of this quantity indicates a stronger concentration of the distribution of the portfolio mortality intensity around its mean, denoting a reduction of longevity risk.

Finally, we introduce the Diversification Index (DI) as an average of the degree of dissimilarity of the mortality intensities of the cohorts in different populations. This measure is a synthetic way of quantifying the level of diversification achieved by a foreign expansion.

\subsection{Percentage risk margin}

To be able to compare the effects of an expansion, we consider first a normalized quantity that is, the ratio of the risk margin and the actuarial value of a portfolio $\Pi$, namely a percentage risk margin:

$$
\% R M_{\Pi}=\frac{R M_{\Pi}(t)}{A V_{\Pi}(t)} .
$$

A lower percentage risk margin denotes a lower percentage loss in the worst-case scenario, relative to the portfolio value. Hence, reducing this measure is beneficial for the company in two respects. First, it indicates a mitigation in the risk connected to adverse scenarios. In this sense, the risk margin can be considered as a measure of the systemic risk that the company may generate, by triggering losses that will hit its creditors. Second, it represents a capital requirement reduction, which frees up resources. Because the risk margin can be interpreted as both a capital requirement and a measure of the loss the company can generate - at a given level of confidence - among its creditors, it is then conceivable that minimizing the percentage risk margin aligns the interests of both the insurance company and its regulators. In what follows, we take the point of view of the insurer, taking for granted the alignment of her interest with the ones of the regulator. 


\subsection{Standard deviation of the portfolio mortality intensity}

Another measure of the diversification effects deriving from longevity risk pooling across populations can be derived by looking at the change in the standard deviation of the portfolio mortality intensity pre- and post-foreign expansion. Given an annuity portfolio $\Pi$, we define its portfolio mortality intensity $\lambda^{\Pi}$ as the weighted average of the mortality intensities of each generation in the portfolio, where the weights are the percentages of contracts written on each generation. Considering the initial domestic portfolio $\Pi^{0}$, let $n_{i}^{d}$ be the number of contracts sold to generation $i$ belonging to the domestic population, and let $n^{d}=\sum_{i=1}^{m} n_{i}^{d}$ be the total number of contracts in the portfolio. Then, we define $\lambda^{\Pi^{0}}$ as

$$
\lambda^{\Pi^{0}}(t)=\sum_{i=1}^{m} \frac{n_{i}^{d}}{n^{d}} \lambda_{i}^{d}(t)=\sum_{i=1}^{m} w_{i}^{d} \lambda_{i}^{d}(t),
$$

where $\omega_{i}^{d}=\frac{n_{i}^{d}}{n^{d}}$ is the weight for each generation $i$ of the domestic population. Similarly, let $n=n^{d}+n^{f}$ be the total number of contracts in the portfolio, $\Pi^{2}$, after a foreign expansion in which $n^{f}$ contracts are written on the target foreign population, $n_{i}^{f}$ on each generation $i$. The mortality intensity of the portfolio $\Pi^{2}$ is given by

$$
\begin{aligned}
\lambda^{\Pi^{2}}(t) & =\sum_{i=1}^{m} \frac{n_{i}^{d}}{n} \lambda_{i}^{d}(t)+\frac{n_{i}^{f}}{n} \lambda_{i}^{f}(t) \\
& =\sum_{i=1}^{m} w_{i}^{d, \Pi^{2}} \lambda_{i}^{d}(t)+\sum_{i=1}^{m} w_{i}^{f, \Pi^{2}} \lambda_{i}^{f}(t),
\end{aligned}
$$

where $w_{i}^{d, \Pi^{2}}=\frac{n_{i}^{d}}{n}$ and $w_{i}^{f, \Pi^{2}}=\frac{n_{i}^{f}}{n}$ represent the weights in the portfolio for each generation of the domestic and foreign population, respectively.

Starting with the initial domestic portfolio $\Pi^{0}$ and its mortality intensity $\lambda^{\Pi^{0}}$ defined in (5.2), we have that

$$
\begin{aligned}
\operatorname{Var}_{0}\left(\lambda^{\Pi^{0}}(t)\right) & =\operatorname{Var}_{0}\left(\sum_{i=1}^{m} w_{i}^{d} \lambda_{i}^{d}(t)\right) \\
& =\sum_{i=1}^{m}\left(w_{i}^{d}\right)^{2} \operatorname{Var}_{0}\left(\lambda_{i}^{d}(t)\right)+2 \sum_{i<j} w_{i}^{d} w_{j}^{d} \operatorname{Cov}_{0}\left(\lambda_{i}^{d}(t), \lambda_{j}^{d}(t)\right) .
\end{aligned}
$$

Thus, we define the standard deviation of the portfolio $\Pi^{0}$ mortality intensity as

$$
\sigma^{\lambda}\left(\Pi^{0}\right)=\sqrt{\operatorname{Var}_{0}\left(\lambda^{\Pi^{0}}(t)\right)}
$$


Similarly, considering the post expansion portfolio $\Pi^{2}: 4$

$$
\sigma^{\lambda}\left(\Pi^{2}\right)=\sqrt{\operatorname{Var}_{0}\left(\lambda \Pi^{2}(t)\right)},
$$

with $\operatorname{Var}_{0}\left(\lambda^{\Pi^{2}}(t)\right)$ defined as in the Appendix. In Appendix A, we also provide a closed-form approximation, obtained using the Gaussian mapping technique, as in Brigo and Mercurio (2001).

A foreign expansion provides a diversification benefit if

$$
\sigma^{\lambda}\left(\Pi^{2}\right)<\sigma^{\lambda}\left(\Pi^{0}\right) \text {. }
$$

This can happen because, after the expansion, $\lambda^{\Pi^{2}}(t)$ depends on $\lambda_{i}^{d}$, but also on the different risk source $\lambda_{i}^{f}$ that may be non perfectly correlated with $\lambda_{i}^{d}$ for $i=1, \ldots, m$.

Moreover, if there are multiple target portfolios for a foreign expansion, a possible way to decide about the optimal expansion target would be to choose the portfolio that provides the lowest $\sigma^{\lambda}\left(\Pi^{*}\right)$.

\subsection{Similarity/Diversification index}

Building up on the characteristics of the longevity model described in the previous section, finally, we propose the Similarity and Diversification index, as follows. Let $n_{i}^{d}$ be the number of annuities written on cohort $x_{i}$ belonging to the domestic population, $n_{i}^{f}$ the number of annuities written on cohort $x_{i}$ belonging to the foreign population, $n_{i}=n_{i}^{d}+n_{i}^{f}$ and $m$ the number of generations in the initial, domestic portfolio. Then the DI is equal to:

$$
D I=\frac{1}{m} \sum_{i=1}^{m} \frac{n_{i}^{f}\left(1-\delta_{i}\right)}{n_{i}},
$$

and the Similarity Index $(\mathrm{SI})^{5}$ is

$$
S I=1-D I .
$$

The DI is model-specific, and it represents a weighted average of the dissimilarities between the same cohorts in different populations, ${ }^{6}$ present in both the initial portfolio and in the portfolio after the expansion. Dissimilarities are captured by the complement to 1 of $\delta_{i}$, the generation-specific parameter that captures the degree of correlation between the same generation of the different populations. The weights, $n_{i}^{f} / n_{i}$, are given, for each cohort in the initial portfolio, by the number of annuities in the foreign population (after the expansion) relative to the total number of annuities written on that cohort in both populations. We average the weighted dissimilarities across all the $m$ cohorts of the domestic population initially present in the annuity portfolio. 
Our proposed indicator has the following properties. First, $0 \leq D I \leq 1$. If $\delta_{i}=1$ for every $i$, that is, the two portfolios are written on perfectly correlated populations, then, obviously, $S I=1$ and $D I=0$. On the other hand, if $\delta_{i}=0$, for every $i$, which means that the intensities of the foreign population are independent of the risk factor of the domestic, the DI does not go to 1 independently of the portfolio composition. If $n_{i}^{f} \rightarrow \infty$ and $n_{i}^{d}$ remains constant, then $S I \rightarrow 0, D I \rightarrow 1$. This happens because the longevity risk of the foreign population is completely idiosyncratic and therefore diversification is reaped only enlarging the foreign portfolio as much as possible. This shows that the DI appropriately reflects both the properties of the intensity correlation structure and the portfolio mix chosen by the underwriter. ${ }^{7}$

Further insights on the properties and indications deriving from the three measures presented will emerge from the application in Section 6, but let us comment briefly on them before going on.

The percentage risk margin has the advantage of being expressed in economic terms, allowing a comparison between the economic benefit of a foreign expansion and its implementation cost, and between the benefits of competing target portfolios. Among the three measures, the percentage risk margin is the only one that can capture the impact of the term structure of interest rates on the economic benefit of geographical longevity risk diversification. However, computing it - for our proposed model, at least - requires Monte Carlo simulations, making it the most computationally expensive measure among the ones presented.

The standard deviation of the portfolio mortality intensity does not require Monte Carlo simulations, if Gaussian mapping is used, and can provide similar information to the risk margin when comparing different expansion strategies. It is able to capture the entire dependence structure between the domestic and foreign generations, but it is simply a distributional property and not a monetary measure.

The DI is the easiest measure to compute because it does not require Monte Carlo simulations or the estimation of a correlation matrix. However, it does not capture the entire dependence structure between the domestic and foreign generations and, therefore, can provide useless indications when the target foreign portfolio population shows low dependence across generations and when the diversification benefit of grouping different cohorts belonging to different populations is large.

\section{Application}

In this section, we calibrate our proposed model and try to quantify the diversification gains deriving from an international expansion toward Italy of an initially UK-based annuity portfolio versus a domestic expansion. Notice that our model is asymmetric in nature: the calibration thus is not insensitive to the choice of the labels of "domestic" and "foreign" populations. In what follows 
TABLE 1

Domestic Population (UK) CALIBRATION RESUlts.

\begin{tabular}{cccccc}
\hline \hline Age & $a$ & $b$ & $\sigma$ & $\lambda_{0}$ & RMSE \\
\hline 65 & $2.7878 \times 10^{-5}$ & 0.0723 & 0.0075 & 0.0116 & 0.00035 \\
66 & $6.5423 \times 10^{-5}$ & 0.0652 & 0.0059 & 0.0124 & 0.00028 \\
67 & $1.8424 \times 10^{-5}$ & 0.0740 & 0.0080 & 0.0135 & 0.00035 \\
68 & $5.3144 \times 10^{-5}$ & 0.0685 & 0.0084 & 0.0160 & 0.00043 \\
69 & $1.2500 \times 10^{-4}$ & 0.0589 & 0.0091 & 0.0164 & 0.00039 \\
70 & $8.4734 \times 10^{-5}$ & 0.0646 & 0.0108 & 0.0189 & 0.00056 \\
71 & $7.1323 \times 10^{-5}$ & 0.0667 & 0.0106 & 0.0212 & 0.00038 \\
72 & $4.1759 \times 10^{-5}$ & 0.0688 & 0.0073 & 0.0239 & 0.00040 \\
73 & $2.2984 \times 10^{-5}$ & 0.0689 & 0.0066 & 0.0262 & 0.00063 \\
74 & $9.6036 \times 10^{-5}$ & 0.0663 & 0.0131 & 0.0282 & 0.00040 \\
75 & $3.3898 \times 10^{-5}$ & 0.0684 & 0.0077 & 0.0316 & 0.00049 \\
\hline \hline
\end{tabular}

we consider the UK population as domestic, which is more natural when depicting the case of an expansion made by a UK-based insurer. However, we obtained very similar (unreported) results when treating the Italian mortality intensities as domestic, implying the robustness of our application outcomes.

\subsection{Mortality intensities estimation}

To calibrate our model, we proceed in two steps. First, we calibrate the parameters of the two intensity processes of the domestic and of the foreign population, respectively. Then, in a second step, we calibrate the correlation parameters $\rho_{i j}$. We calibrate the parameters of the mortality model to the generations of UK and Italian males whose age, at 31/12/2012, is between 65 and 75, that is, the cohorts born between 1937 and 1947. We consider thus 11 different cohorts present in the initial portfolio: $x_{i}=65, \ldots, 75$. We use the 1-year $\times$ 1-year cohort death rates data provided by the Human Mortality Database and recover, using the 20 observations from 1993 and $2012^{8}$ the observed conditional survival probabilities, for each cohort, for the individuals alive in 1993. The estimation of the parameters is performed minimizing the Rooted Mean Squared Error (RMSE) between the observed and the model-implied survival probabilities. Tables 1 and 2 report the calibrated parameters for the two populations, while Figures 1 and 2 report the actual and fitted survival probabilities and the calibration errors, respectively. In particular, we show in Tables 3 and 4 the mean absolute percentage error (MAPE) of the model by age and year for the two populations. The model, although parsimonious (it requires the estimation of $4^{*} \mathrm{M}+6$ parameters, versus the $5^{*} \mathrm{M}+3$ parameters estimated by Yang and Wang (2013), with $M$ number of ages considered), is able to capture remarkably well the survival probability curves of the two populations, for all the cohorts considered, with an average absolute percentage errors by age as low as less than $0.08 \%$ for all the ages considered. 
TABLE 2

Foreign Population (IT) CALIBRATION RESUlts.

\begin{tabular}{|c|c|c|c|c|c|c|}
\hline Age & $a^{\prime}$ & $b^{\prime}$ & $\sigma^{\prime}$ & $\delta$ & RMSE & $\lambda_{0}^{\prime}$ \\
\hline 65 & & & & 0.7939 & 0.00045 & \\
\hline 66 & & & & 0.8528 & 0.00017 & \\
\hline 67 & & & & 0.9369 & 0.00038 & \\
\hline 68 & & & & 0.8289 & 0.00036 & \\
\hline 69 & & & & 0.9039 & 0.00045 & \\
\hline 70 & $1.1785 \times 10^{-4}$ & $4.7825 \times 10^{-7}$ & 0.0153 & 0.8362 & 0.00032 & 0.0022 \\
\hline 71 & & & & 0.8548 & 0.00034 & \\
\hline 72 & & & & 0.8210 & 0.00036 & \\
\hline 73 & & & & 0.8203 & 0.00036 & \\
\hline 74 & & & & 0.8484 & 0.00071 & \\
\hline 75 & & & & 0.8683 & 0.00078 & \\
\hline
\end{tabular}
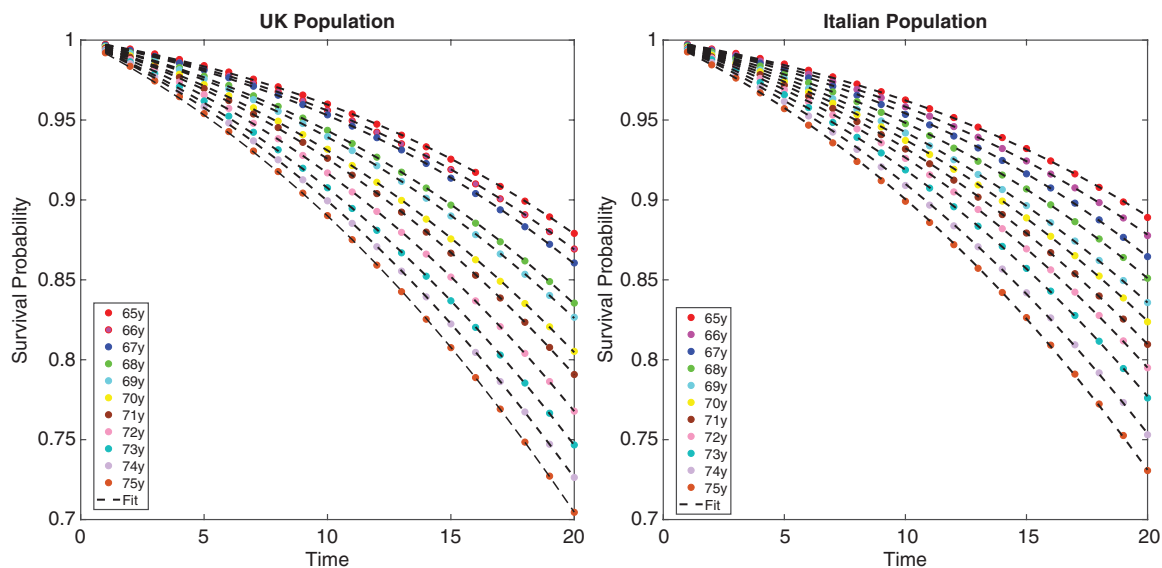

FIGURE 1: Observed and theoretical survival probabilities. The left panel shows the observed versus fitted survival probabilities for the foreign population, while the right reports the figures for the domestic population.
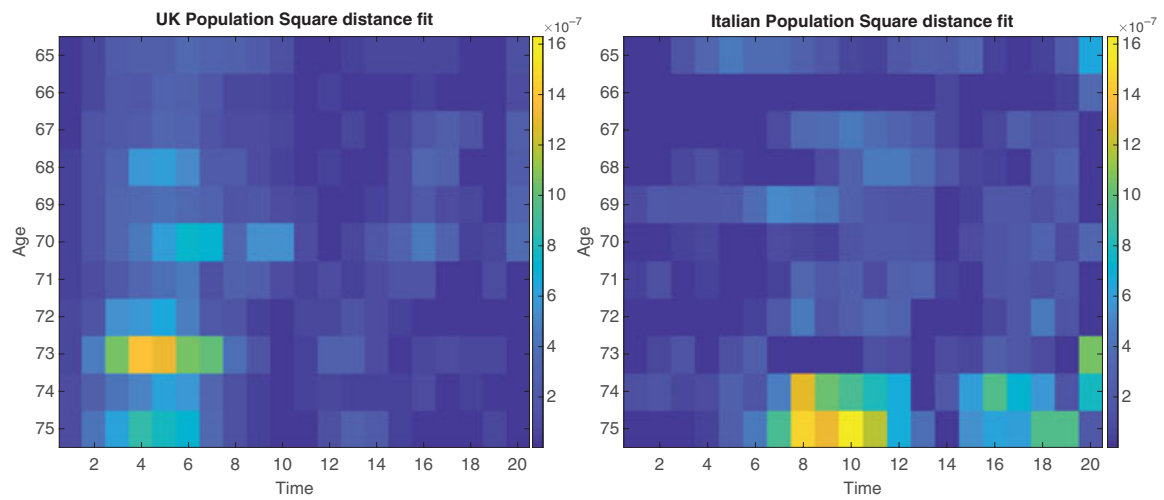

FIGURE 2: Calibration errors. 
TABLE 3

MeAn Absolute PERCENTAge ERror by AGE.

\begin{tabular}{cccccccccccc}
\hline \hline & 65 & 66 & 67 & 68 & 69 & 70 & 71 & 72 & 73 & 74 & 75 \\
\hline UK & $0.03 \%$ & $0.02 \%$ & $0.03 \%$ & $0.04 \%$ & $0.04 \%$ & $0.06 \%$ & $0.04 \%$ & $0.03 \%$ & $0.06 \%$ & $0.04 \%$ & $0.04 \%$ \\
ITA & $0.04 \%$ & $0.01 \%$ & $0.03 \%$ & $0.03 \%$ & $0.04 \%$ & $0.03 \%$ & $0.03 \%$ & $0.03 \%$ & $0.03 \%$ & $0.07 \%$ & $0.08 \%$ \\
\hline \hline
\end{tabular}

\subsection{Correlation matrix estimation}

After having estimated the cohort-specific parameters of the two populations, we turn to the estimation of their correlation structure. Having chosen a nonGaussian process for the mortality intensities of the cohorts, we are not able to derive a formula for their correlations in closed form. However, to estimate correlations, we can apply the Gaussian Mapping technique, proposed in Brigo and Mercurio (2001) and Brigo and Alfonsi (2005), and described in Appendix A. This gives an approximation, which however has been proven to be accurate in similar contexts, ${ }^{9}$ as in Brigo and Alfonsi (2005). Such technique allows to obtain a closed-form approximation of the correlations between the intensities of the different cohorts by mapping a CIR process into a Vasicek process that is as close as possible to the original one, in the sense that it has the same survival probabilities. The correlation between the CIR processes $\lambda_{i}^{d}(t)$ and $\lambda_{j}^{d}(t)$ is approximated by the correlation between the Vasicek processes $\lambda_{i}^{V}(t)$ and $\lambda_{j}^{V}(t)$, defined in Appendix A:

$$
\begin{aligned}
\operatorname{Corr}_{0}\left(\lambda_{i}^{d}(t), \lambda_{j}^{d}(t)\right) \approx \operatorname{Corr}_{0}\left(\lambda_{i}^{V}(t), \lambda_{j}^{V}(t)\right) & =\frac{\operatorname{Cov}_{0}\left(\lambda_{i}^{V}(t), \lambda_{j}^{V}(t)\right)}{\sqrt{\operatorname{Var}_{0}\left[\lambda_{i}^{V}(t)\right] \operatorname{Var}_{0}\left[\lambda_{j}^{V}(t)\right]}} \\
& =\frac{2 \rho_{i j}}{b_{i}+b_{j}} \cdot \frac{e^{\left(b_{i}+b_{j}\right) t}-1}{\sqrt{\frac{\left(e^{2} b_{i} t-1\right)\left(e^{2} b_{j} t-1\right)}{b_{i} b_{j}}}} .
\end{aligned}
$$

To estimate the correlation parameters, first the parameters of the process described by (A.4) are recovered. Then, using the central mortality rates data available in the UK life tables, ${ }^{10}$ we estimate the instantaneous correlations $\rho_{i j}$ between $d \lambda_{i}$ and $d \lambda_{j}$ by inverting the approximated correlation expression (6.1). To compute the correlations between the 11 cohorts involved, we start from the central mortality rates in 1968 of the people aged between 1 and 11, and we follow the diagonal of the life table until we reach the central mortality rates of the people aged between 65 and 75 years in 2012 . The central mortality rates table constructed this way has dimension $65 \times 11$ and allows to estimate the correlation coefficients which we report in Table 5. The upper and lower confidence bounds are computed with bootstrapping from 10,000 resampled samples with replacement. Each sample has dimension $65 \times 11$ and is obtained by randomly choosing 65 times with replacement a row of our original central mortality table. As expected, because of the similarity between the UK and the Italian populations, correlations are close to 1 with tight $95 \%$ confidence 


\section{TABLE 4}

Mean absolute percentage error by time.

\begin{tabular}{|c|c|c|c|c|c|c|c|c|c|c|c|c|c|c|c|c|c|c|c|c|}
\hline & 1 & 2 & 3 & 4 & 5 & 6 & 7 & 8 & 9 & 10 & 11 & 12 & 13 & 14 & 15 & 16 & 17 & 18 & 19 & 20 \\
\hline UK & $0.02 \%$ & $0.04 \%$ & $0.06 \%$ & $0.07 \%$ & $0.08 \%$ & $0.07 \%$ & $0.06 \%$ & $0.04 \%$ & $0.04 \%$ & $0.03 \%$ & $0.02 \%$ & $0.02 \%$ & $0.03 \%$ & $0.03 \%$ & $0.03 \%$ & $0.04 \%$ & $0.03 \%$ & $0.02 \%$ & $0.02 \%$ & $0.03 \%$ \\
\hline ITA & $0.01 \%$ & $0.02 \%$ & $0.02 \%$ & $0.02 \%$ & $0.03 \%$ & $0.03 \%$ & $0.04 \%$ & $0.05 \%$ & $0.05 \%$ & $0.06 \%$ & $0.06 \%$ & $0.05 \%$ & $0.04 \%$ & $0.04 \%$ & $0.05 \%$ & $0.05 \%$ & $0.05 \%$ & $0.05 \%$ & $0.05 \%$ & $0.05 \%$ \\
\hline
\end{tabular}

$\begin{array}{lllllllllllllllllllll}\text { ITA } & 0.01 \% & 0.02 \% & 0.02 \% & 0.02 \% & 0.03 \% & 0.03 \% & 0.04 \% & 0.05 \% & 0.05 \% & 0.06 \% & 0.06 \% & 0.05 \% & 0.04 \% & 0.04 \% & 0.05 \% & 0.05 \% & 0.05 \% & 0.05 \% & 0.05 \% & 0.05 \%\end{array}$ 
TABLE 5

INSTANTANEOUS CORRELATION MATRIX UK POPULATION WITH UPPER AND LOWER 95\% CONFIDENCE BOUNDS FROM BOOSTRAPPING.

\begin{tabular}{|c|c|c|c|c|c|c|c|c|c|c|c|}
\hline & 65 & 66 & 67 & 68 & 69 & 70 & 71 & 72 & 73 & 74 & 75 \\
\hline UB & & (0.99938) & (0.999000) & $(0.998716)$ & $(0.998124)$ & $(0.997751)$ & $(0.997741)$ & $(0.997332)$ & $(0.997197)$ & (0.99709) & $(0.996878)$ \\
\hline 65 & 1 & 0.996614 & 0.993845 & 0.992351 & 0.990865 & 0.989791 & 0.988837 & 0.987733 & 0.987419 & 0.987183 & 0.986708 \\
\hline LB & & $(0.9909)$ & $(0.983627)$ & $(0.980001)$ & $(0.977076)$ & $(0.974617)$ & $(0.97186)$ & $(0.969869)$ & $(0.968956)$ & $(0.968354)$ & $(0.966995)$ \\
\hline UB & $(0.999378)$ & & $(0.999558)$ & $(0.999358)$ & $(0.999023)$ & $(0.99862)$ & $(0.998523)$ & $(0.998186)$ & $(0.998116)$ & $(0.998188)$ & $(0.997826)$ \\
\hline 66 & 0.996614 & 1 & 0.999089 & 0.998491 & 0.997805 & 0.99704 & 0.996548 & 0.995866 & 0.995682 & 0.995693 & 0.995174 \\
\hline LB & $(0.991186)$ & & (0.998287) & $(0.997043)$ & $(0.995688)$ & $(0.994296)$ & $(0.993232)$ & $(0.991792)$ & $(0.991525)$ & $(0.991261)$ & $(0.990592)$ \\
\hline UB & $(0.999017)$ & $(0.999559)$ & & $(0.999578)$ & $(0.999456)$ & (0.999219) & $(0.999085)$ & $(0.998907)$ & $(0.998915)$ & $(0.998813)$ & $(0.998598)$ \\
\hline 67 & 0.993845 & 0.999089 & 1 & 0.999317 & 0.999113 & 0.998707 & 0.998423 & 0.998021 & 0.997997 & 0.997849 & 0.997468 \\
\hline LB & $(0.983628)$ & $(0.998268)$ & & $(0.999077)$ & $(0.998673)$ & $(0.997955)$ & $(0.997325)$ & $(0.996572)$ & $(0.996455)$ & $(0.996298)$ & $(0.995615)$ \\
\hline UB & $(0.998679)$ & $(0.999353)$ & (0.99958) & & $(0.99971)$ & $(0.999449)$ & $(0.999293)$ & $(0.999087)$ & $(0.999063)$ & $(0.999027)$ & $(0.998571)$ \\
\hline 68 & 0.992351 & 0.998491 & 0.999317 & 1 & 0.999553 & 0.999168 & 0.998931 & 0.998549 & 0.99852 & 0.998456 & 0.997807 \\
\hline LB & $(0.979943)$ & $(0.997032)$ & $(0.999073)$ & & $(0.999417)$ & $(0.998807)$ & $(0.998385)$ & $(0.997821)$ & $(0.997697)$ & $(0.997582)$ & $(0.99674)$ \\
\hline UB & $(0.998116)$ & (0.99904) & $(0.999447)$ & $(0.99971)$ & & $(0.999733)$ & $(0.999568)$ & (0.999369) & $(0.999376)$ & $(0.999438)$ & $(0.9989)$ \\
\hline 69 & 0.990865 & 0.997805 & 0.999113 & 0.999553 & 1 & 0.999569 & 0.999189 & 0.999009 & 0.999047 & 0.999114 & 0.998333 \\
\hline LB & $(0.97749)$ & $(0.995701)$ & $(0.998669)$ & $(0.999414)$ & & $(0.999343)$ & $(0.998844)$ & $(0.998593)$ & $(0.998596)$ & $(0.998586)$ & $(0.997635)$ \\
\hline
\end{tabular}


TABle 5

Continued.

\begin{tabular}{|c|c|c|c|c|c|c|c|c|c|c|c|}
\hline & 65 & 66 & 67 & 68 & 69 & 70 & 71 & 72 & 73 & 74 & 75 \\
\hline UB & $(0.997754)$ & (0.998619) & $(0.999221)$ & $(0.999444)$ & $(0.999732)$ & & $(0.999625)$ & $(0.99954)$ & $(0.999555)$ & $(0.999654)$ & $(0.999236)$ \\
\hline 70 & 0.989791 & 0.99704 & 0.998707 & 0.999168 & 0.999569 & 1 & 0.999263 & 0.99927 & 0.999281 & 0.999448 & 0.998791 \\
\hline LB & $(0.974657)$ & $(0.99433)$ & $(0.998)$ & $(0.998816)$ & $(0.999344)$ & & $(0.998901)$ & $(0.998917)$ & $(0.998931)$ & (0.999094) & $(0.998258)$ \\
\hline UB & $(0.997714)$ & $(0.998512)$ & $(0.999078)$ & $(0.999293)$ & $(0.999566)$ & $(0.999631)$ & & $(0.999724)$ & $(0.999777)$ & $(0.999767)$ & $(0.999632)$ \\
\hline 71 & 0.988837 & 0.996548 & 0.998423 & 0.998931 & 0.999189 & 0.999263 & 1 & 0.999609 & 0.999689 & 0.999442 & 0.999444 \\
\hline LB & $(0.972173)$ & $(0.993218)$ & $(0.997347)$ & $(0.998366)$ & $(0.998853)$ & $(0.998884)$ & & $(0.999512)$ & $(0.999578)$ & $(0.999165)$ & $(0.999215)$ \\
\hline UB & $(0.997296)$ & $(0.998214)$ & $(0.998897)$ & (0.999096) & $(0.999366)$ & $(0.999541)$ & $(0.9997259$ & & $(0.999917)$ & $(0.999875)$ & $(0.999819)$ \\
\hline 72 & 0.969828 & 0.991711 & 0.996482 & 0.997807 & 0.99859 & 0.998918 & 0.999511 & 1 & 0.999779 & 0.999616 & 0.999536 \\
\hline LB & & $(0.9909)$ & $(0.983627)$ & $(0.980001)$ & $(0.977076)$ & $(0.974617)$ & $(0.97186)$ & $(0.969869)$ & $(0.968956)$ & $(0.968354)$ & $(0.966995)$ \\
\hline UB & $(0.997148)$ & $(0.998104)$ & (0.99892) & (0.999065) & $(0.999377)$ & $(0.999555)$ & (0.999777) & (0.999919) & & $(0.9999)$ & $(0.999822)$ \\
\hline 73 & 0.987419 & 0.995682 & 0.997997 & 0.99852 & 0.999047 & 0.999281 & 0.999689 & 0.999862 & 1 & 0.999732 & 0.999698 \\
\hline LB & $(0.969095)$ & $(0.991471)$ & $(0.996389)$ & $(0.997707)$ & $(0.998618)$ & $(0.998915)$ & $(0.999575)$ & (0.99978) & & $(0.999586)$ & (0.99955) \\
\hline UB & $(0.997123)$ & $(0.998201)$ & $(0.998815)$ & (0.999026) & $(0.999433)$ & $(0.999655)$ & $(0.999763)$ & $(0.999876)$ & (0.999898) & & $(0.999815)$ \\
\hline 74 & 0.987183 & 0.995693 & 0.997849 & 0.998456 & 0.999114 & 0.999448 & 0.999442 & 0.999746 & 0.999732 & 1 & 0.999547 \\
\hline LB & $(0.968577)$ & $(0.991343)$ & $(0.996281)$ & $(0.997581)$ & $(0.998579)$ & $(0.999085)$ & $(0.999171)$ & $(0.999618)$ & $(0.999585)$ & & (0.99928) \\
\hline UB & $(0.996825)$ & (0.997807) & (0.998599) & $(0.998586)$ & $(0.998896)$ & $(0.999231)$ & $(0.999633)$ & (0.999817) & (0.999819) & $(0.999811)$ & \\
\hline 75 & 0.986708 & 0.995174 & 0.997468 & 0.997807 & 0.998333 & 0.998791 & 0.999444 & 0.999701 & 0.999698 & 0.999547 & 1 \\
\hline LB & $(0.968182)$ & (0.99056) & $(0.995587)$ & $(0.9967)$ & $(0.997637)$ & $(0.998261)$ & $(0.999215)$ & $(0.999532)$ & $(0.999552)$ & $(0.999285)$ & \\
\hline
\end{tabular}


bounds, but they tend to decrease with the distance between the initial ages of the two considered cohorts. This behavior aligns with the intuition that the changes leading to longevity improvements (such as healthy habits or medical advancements) have different impact on different generations and that cohort effects are at play. Table 6 reports instead the correlations across the two populations. Also in this case, the correlations appearing in the diagonals are the highest, and they tend to decrease along the rows and column dimensions, indicating the presence of common cohort effects across populations. Figure 3 shows the covariances between the different UK cohorts and between the UK and Italian cohorts. The two generations with the lowest covariance are the 66-year-old UK and 66-year-old Italian cohorts. Both the UK and Italian 66-year-old cohorts are the ones with the lowest covariance with all other generations in the other country.

\subsection{Evaluating the diversification gains in terms of risk margin}

Because the oldest cohort considered in our application is 75 , and we assume a maximum life span of $\omega=105$ years, we fix the time horizon of our simulations to 30 years. Consistent with this choice, we consider a constant interest rate of $2 \%$, matching the 30-year risk-free-rate indicated by EIOPA for the calculation of technical provisions. The choice of a constant interest rate term structure allows us to isolate and capture any possible added benefit specifically due to the geographical diversification of an annuity portfolio. The time horizon at which the Risk Margin is computed is 15 years. This choice is justified because we want to focus on the medium-long term benefits of geographical diversification. Consistently with the Solvency II regulation, we select a confidence level $\alpha=99.5 \%$ when calculating the Risk Margin associated to the portfolio.

\section{Initial Portfolio}

The UK Insurer has an initial portfolio $\Pi^{0}$ made of 1000 contracts sold to males whose age, at 31/12/2012, is between 65 and 75 years. The distribution of contracts among ages reflects the proportions of individuals aged between 65 and 75 years in the UK national population. For instance, since in the general UK population 69 years old constitute $11.00 \%$ of all the people aged between 65 and 75 years, the domestic portfolio contains 110 contracts sold to 69 years old (see Table 7).

The initial Actuarial Value $A V_{\Pi^{0}}(0)$ of the portfolio is

$$
A V_{\Pi^{0}}(0)=1.4104 \times 10^{4},
$$

while the Risk Margin computed at time 0 is

$$
R M_{\Pi^{0}}(0)=1.1838 \times 10^{3} .
$$


TABLE 6

CORRELATION BETWEEN POPULATIONS WITH UPPER AND LOWER 95\% CONFIDENCE BOUNDS FROM BOOSTRAPPING. ROWS ARE UK GENERATIONS WHILE COLUMNS ARE ITA GENERATIONS.

\begin{tabular}{|c|c|c|c|c|c|c|c|c|c|c|c|}
\hline & 65 & 66 & 67 & 68 & 69 & 70 & 71 & 72 & 73 & 74 & 75 \\
\hline UB & $(0.958536)$ & $(0.979156)$ & $(0.980102)$ & $(0.97169)$ & $(0.972043)$ & $(0.97753)$ & $(0.976328)$ & (0.978398) & $(0.970923)$ & $(0.977916)$ & $(0.963227)$ \\
\hline 65 & 0.958536 & 0.976454 & 0.97503 & 0.965521 & 0.964995 & 0.969704 & 0.967647 & 0.968971 & 0.961354 & 0.968185 & 0.953476 \\
\hline LB & $(0.958536)$ & $(0.971023)$ & $(0.965148)$ & $(0.953324)$ & $(0.951457)$ & $(0.955025)$ & $(0.950993)$ & $(0.951074)$ & $(0.943368)$ & $(0.950217)$ & $(0.934664)$ \\
\hline UB & $(0.958504)$ & $(0.980357)$ & $(0.981229)$ & $(0.972916)$ & $(0.973531)$ & $(0.978934)$ & $(0.977709)$ & $(0.979876)$ & $(0.972373)$ & $(0.979556)$ & $(0.964782)$ \\
\hline 66 & 0.955861 & 0.980357 & 0.980761 & 0.972075 & 0.972336 & 0.97739 & 0.975777 & 0.977536 & 0.969978 & 0.977114 & 0.962233 \\
\hline LB & $(0.950595)$ & $(0.980357)$ & $(0.979968)$ & $(0.970672)$ & $(0.970352)$ & $(0.974748)$ & $(0.972428)$ & $(0.973557)$ & $(0.965942)$ & $(0.972905)$ & $(0.957943)$ \\
\hline UB & $(0.958127)$ & (0.9799) & $(0.98163)$ & $(0.973106)$ & $(0.973914)$ & (0.979498) & $(0.978242)$ & (0.980498) & $(0.973106)$ & (0.980139) & $(0.965516)$ \\
\hline 67 & 0.953179 & 0.979438 & 0.98163 & 0.972854 & 0.973585 & 0.978999 & 0.977588 & 0.979627 & 0.972208 & 0.979203 & 0.964426 \\
\hline LB & $(0.943524)$ & $(0.978657)$ & $(0.98163)$ & $(0.972614)$ & $(0.97315)$ & $(0.978292)$ & $(0.976524)$ & $(0.97819)$ & $(0.970704)$ & $(0.977606)$ & $(0.962632)$ \\
\hline UB & $(0.957858)$ & (0.979697) & $(0.98122)$ & $(0.973526)$ & $(0.974171)$ & $(0.979732)$ & $(0.978443)$ & $(0.980683)$ & $(0.973254)$ & $(0.980351)$ & $(0.965512)$ \\
\hline 68 & 0.951754 & 0.978859 & 0.980966 & 0.973526 & 0.97402 & 0.979458 & 0.978092 & 0.980151 & 0.972725 & 0.979807 & 0.964761 \\
\hline LB & $(0.940025)$ & $(0.977399)$ & $(0.980725)$ & $(0.973526)$ & $(0.97388)$ & (0.979109) & $(0.977554)$ & $(0.979404)$ & $(0.971887)$ & $(0.978966)$ & $(0.963694)$ \\
\hline UB & $(0.957178)$ & (0.979304) & $(0.981013)$ & $(0.973156)$ & $(0.974367)$ & $(0.979922)$ & $(0.978629)$ & $(0.980861)$ & $(0.973472)$ & $(0.980681)$ & $(0.965729)$ \\
\hline 69 & 0.950242 & 0.978098 & 0.980677 & 0.973002 & 0.974367 & 0.979762 & 0.978255 & 0.980513 & 0.97315 & 0.980363 & 0.965181 \\
\hline LB & $(0.936819)$ & $(0.976141)$ & $(0.980238)$ & $(0.972864)$ & $(0.974367)$ & $(0.979541)$ & $(0.977932)$ & $(0.980107)$ & $(0.972719)$ & $(0.979836)$ & $(0.964518)$ \\
\hline UB & $(0.956916)$ & (0.978959) & $(0.980822)$ & $(0.972937)$ & $(0.974142)$ & $(0.980224)$ & $(0.978725)$ & (0.981078) & $(0.973685)$ & $(0.980934)$ & $(0.966091)$ \\
\hline 70 & 0.949249 & 0.977386 & 0.980317 & 0.972666 & 0.973986 & 0.980224 & 0.978367 & 0.98081 & 0.973417 & 0.980729 & 0.965663 \\
\hline LB & $(0.934764)$ & $(0.974749)$ & $(0.979582)$ & $(0.972317)$ & $(0.973764)$ & $(0.980224)$ & $(0.978001)$ & $(0.980467)$ & $(0.973071)$ & $(0.980377)$ & $(0.965158)$ \\
\hline
\end{tabular}


TABLE 6

CONTINUED.

\begin{tabular}{lcccccccccccc}
\hline \hline & 65 & 66 & 67 & 68 & 69 & 70 & 71 & 72 & 73 & 74 & 75 \\
\hline UB & $(0.956931)$ & $(0.978951)$ & $(0.980825)$ & $(0.972915)$ & $(0.974108)$ & $(0.979987)$ & $(0.979213)$ & $(0.981381)$ & $(0.974022)$ & $(0.981165)$ & $(0.966604)$ \\
71 & 0.948454 & 0.977027 & 0.980163 & 0.972558 & 0.973739 & 0.979625 & 0.979213 & 0.981268 & 0.973937 & 0.980846 & 0.966417 \\
LB & $(0.932229)$ & $(0.973718)$ & $(0.979085)$ & $(0.972032)$ & $(0.973406)$ & $(0.979262)$ & $(0.979213)$ & $(0.981174)$ & $(0.973829)$ & $(0.980572)$ & $(0.966199)$ \\
& & & & & & & & & & & & \\
UB & $(0.956826)$ & $(0.978961)$ & $(0.980921)$ & $(0.973006)$ & $(0.97419)$ & $(0.980186)$ & $(0.979232)$ & $(0.981944)$ & $(0.974446)$ & $(0.981556)$ & $(0.967063)$ \\
72 & 0.947671 & 0.976645 & 0.980056 & 0.972471 & 0.973848 & 0.97992 & 0.979119 & 0.981944 & 0.974391 & 0.981429 & 0.96695 \\
LB & $(0.930941)$ & $(0.972758)$ & $(0.978568)$ & $(0.971745)$ & $(0.973446)$ & $(0.979576)$ & $(0.979026)$ & $(0.981944)$ & $(0.97431)$ & $(0.9813)$ & $(0.966792)$ \\
& & & & & & & & & & & & \\
UB & $(0.956183)$ & $(0.978327)$ & $(0.980331)$ & $(0.972373)$ & $(0.973617)$ & $(0.979602)$ & $(0.978686)$ & $(0.981261)$ & $(0.973933)$ & $(0.980987)$ & $(0.966475)$ \\
73 & 0.946796 & 0.975871 & 0.979435 & 0.971852 & 0.973294 & 0.979335 & 0.978599 & 0.981204 & 0.973933 & 0.980824 & 0.966357 \\
LB & $(0.928455)$ & $(0.971803)$ & $(0.977957)$ & $(0.971019)$ & $(0.97287)$ & $(0.978985)$ & $(0.978489)$ & $(0.981125)$ & $(0.973933)$ & $(0.980677)$ & $(0.966211)$ \\
& & & & & & & & & & & \\
UB & $(0.955689)$ & $(0.977943)$ & $(0.979815)$ & $(0.971933)$ & $(0.973264)$ & $(0.979293)$ & $(0.978265)$ & $(0.980801)$ & $(0.973428)$ & $(0.980679)$ & $(0.96606)$ \\
74 & 0.946176 & 0.975475 & 0.97888 & 0.971384 & 0.972952 & 0.979088 & 0.977947 & 0.980676 & 0.973266 & 0.980679 & 0.965806 \\
LB & $(0.928609)$ & $(0.971309)$ & $(0.977319)$ & $(0.970554)$ & $(0.972423)$ & $(0.978737)$ & $(0.977676)$ & $(0.980553)$ & $(0.973122)$ & $(0.980679)$ & $(0.965556)$ \\
& & & & & & & & & & \\
UB & $(0.955982)$ & $(0.978132)$ & $(0.980179)$ & $(0.972063)$ & $(0.973293)$ & $(0.979438)$ & $(0.978696)$ & $(0.981318)$ & $(0.973913)$ & $(0.981055)$ & $(0.966801)$ \\
75 & 0.946262 & 0.975526 & 0.97907 & 0.971311 & 0.97275 & 0.979008 & 0.978514 & 0.981203 & 0.973792 & 0.980795 & 0.966801 \\
LB & $(0.927589)$ & $(0.971166)$ & $(0.977298)$ & $(0.970242)$ & $(0.972053)$ & $(0.978498)$ & $(0.978285)$ & $(0.981041)$ & $(0.97364)$ & $(0.980536)$ & $(0.966801)$ \\
\hline \hline
\end{tabular}


TABLE 7

DOMESTIC PORTFOLIO COMPOSITION.

\begin{tabular}{lccccccccccc}
\hline \hline & 65 & 66 & 67 & 68 & 69 & 70 & 71 & 72 & 73 & 74 & 75 \\
\hline $\begin{array}{l}\% \text { in the } \\
\text { national } \\
\text { population }\end{array}$ & 9.68 & 9.79 & 9.98 & 10.34 & 11.00 & 10.10 & 10.10 & 8.43 & 8.10 & 7.36 & 6.56 \\
$\Pi^{0}$ & 97 & 98 & 100 & 103 & 110 & 101 & 86 & 84 & 81 & 74 & 66 \\
\hline \hline
\end{tabular}

TABLE 8

FOREIGN PORTFOLIO COMPOSITION.

\begin{tabular}{lccccccccccc}
\hline \hline & 65 & 66 & 67 & 68 & 69 & 70 & 71 & 72 & 73 & 74 & 75 \\
\hline $\begin{array}{l}\% \text { in the } \\
\text { national } \\
\text { population }\end{array}$ & 10.31 & 10.73 & 10.48 & 10.39 & 8.01 & 8.18 & 8.26 & 8.09 & 8.09 & 8.86 & 8.60 \\
$\Pi^{F}$ & & & & & & & & & & & \\
\hline \hline
\end{tabular}
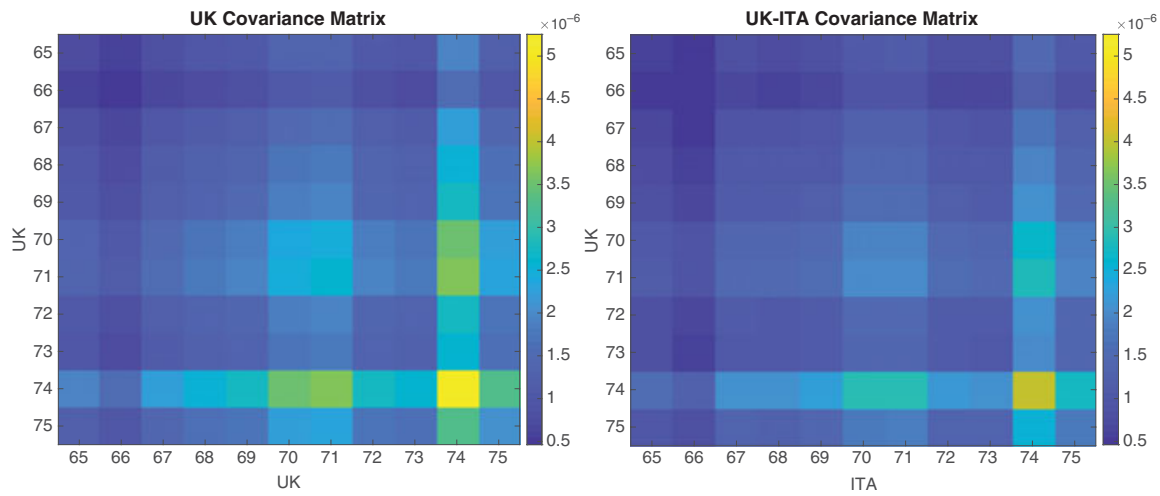

FIGURE 3: Left Panel: Covariance matrix between UK generations. Right Panel: Covariance matrix between Italian and UK generations.

Hence, the initial portfolio value is

$$
\Pi^{0}(0)=A V_{\Pi^{0}}(0)+R M_{\Pi^{0}}(0)=1.5288 \times 10^{4} .
$$

The Risk Margin accounts for $8.39 \%$ of the initial portfolio Actuarial Value and $\sigma^{\lambda}\left(\Pi^{0}\right)=0.00124$.

Portfolio $\Pi^{F}$ is exposed to the foreign population only, distributed among ages according to Table 8, useful for comparison. As we did for the initial portfolio, we assume that the policyholders' distribution reflects the proportion of individuals belonging to each generation between 65 and 75 in the Italian population (see Table 8). Figure 4 shows the different percentage of 
individuals per cohort in the UK and Italian population. For the foreign portfolio $\Pi^{F}$, the risk margin is $7.39 \%$ and $\sigma^{\lambda}\left(\Pi^{F}\right)=0.00107$. One could guess that by expanding toward Italy, the UK underwriter could, at most, reduce his risk margin to this level. However, we will show later on that, thanks to the diversification effect, the risk margin of the underwriter can be even lower.

\section{Domestic Expansion}

With a domestic expansion, we assume that the Insurer doubles the size of her annuity portfolio, selling additional policies to her domestic population, that is, the UK population. The new portfolio $\Pi^{1}$ is therefore composed of 2000 contracts and is obtained by simply doubling the number of contracts for each generation. Hence,

$$
\begin{aligned}
A V_{\Pi^{1}}(0) & =2.8208 \times 10^{4}, \\
R M_{\Pi^{1}}(0) & =2.3676 \times 10^{3}, \\
\Pi^{1}(0) & =3.0576 \times 10^{4} .
\end{aligned}
$$

The Risk Margin proportion relative to actuarial value is unaffected by the size of the portfolio and still accounts for $8.39 \%$ of the actuarial value of the domestically expanded portfolio. Similarly, also the portfolio mortality standard deviation remains unchanged. In this case, the diversification index between $\Pi^{0}$ and $\Pi^{1}-\Pi^{0}$ is 0 , as no diversification gain can be obtained. However, some diversification gains could be obtained through a domestic expansion, in case the new portfolio had a different composition, in terms of policyholders' ages, than the initial one.

\section{Foreign Expansion}

In case of a Foreign Expansion, we assume that the Insurer doubles the number of policies in its annuity portfolio by selling contracts written on policyholders belonging to the foreign population. The composition of the foreign portfolio per cohort is assumed to follow the same proportions of the Italian population $^{11}$ (see Figure 4). The new portfolio $\Pi^{2}$ is, therefore, composed of 1000 contracts sold to the UK population composing the initial portfolio and of 1000 contracts written on the Italian population, $\Pi^{2}=\Pi^{0}+\Pi^{F}$ (both distributed as described in Table 7). It has the following actuarial value and risk margin:

$$
\begin{aligned}
A V_{\Pi^{2}}(0) & =2.8872 \times 10^{4}, \\
R M_{\Pi^{2}}(0) & =2.2749 \times 10^{3},
\end{aligned}
$$

As a consequence,

$$
\Pi^{2}(0)=3.1147 \times 10^{4}
$$

For this portfolio, the Risk Margin accounts for $7.87 \%$ of the Actuarial Value, reduced, as expected, by 0.52 percentage points relative to the one of 


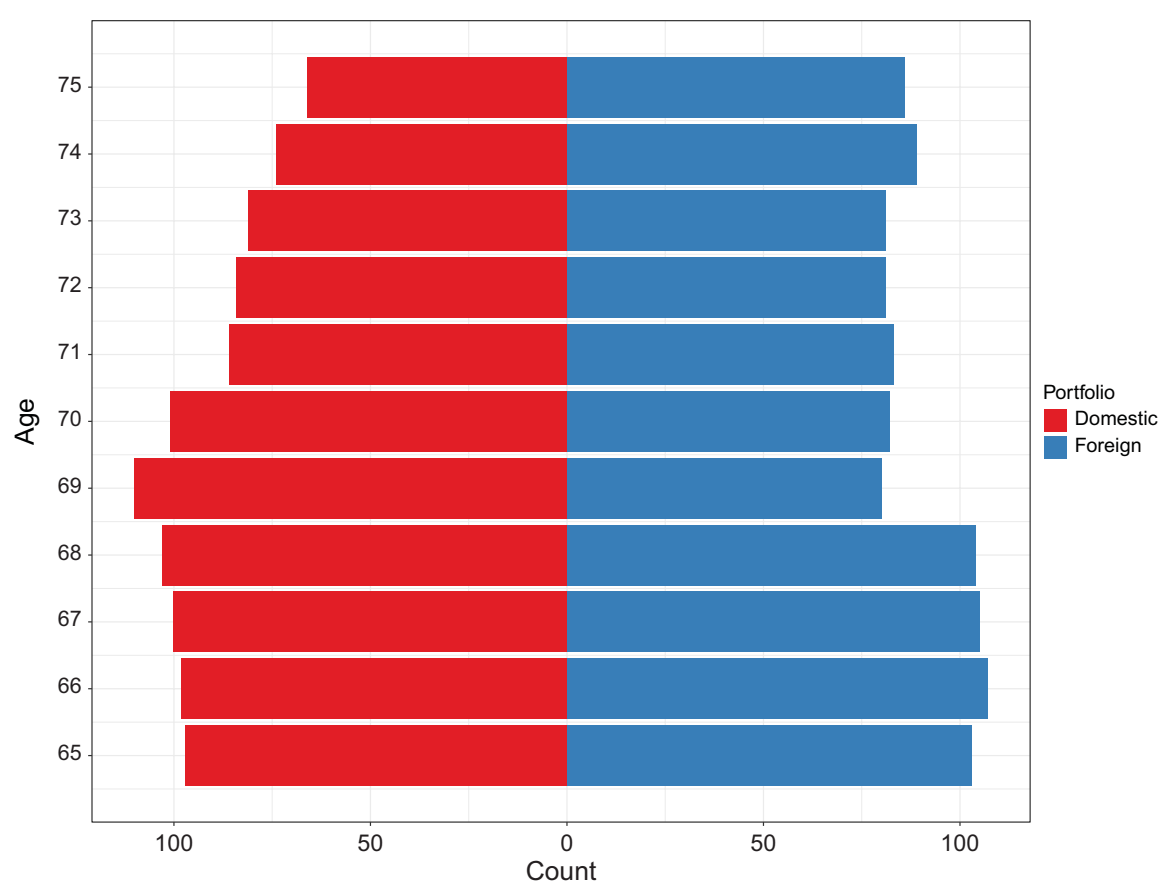

FIGURE 4: Domestic and foreign portfolio composition.

the initial portfolio. The portfolio mortality standard deviation $\sigma^{\lambda}\left(\Pi^{2}\right)$ consistently decreases to 0.00115 , and the diversification index increases to 0.0746 . The diversification gain provided by the Foreign portfolio just described can be further exploited. We then explore alternative portfolios and summarize the results in terms of actuarial values, risk margins and total values in Table 9.

Portfolio $\Pi^{3}$ represents a more aggressive foreign expansion, where the number of policies sold to each generation of foreign policyholders is twice the number of policies in $\Pi^{F}$. Tilting the portfolio towards the foreign population has the effect of decreasing the percentage risk margin $(7.71 \%)$ and the portfolio mortality standard deviation (0.00112), while increasing the diversification index (0.0992). However, it is evident that, at most, by increasing the exposure to the Italian population, the risk margin can not be lower than $7.39 \%$, which is the risk margin of the Foreign portfolio. This suggests to optimize the portfolio mix using not only the diversification across populations, but also across generations.

The portfolio $\Pi_{o p t}^{1}$ is obtained diversifying within the UK population. Its composition is optimized to obtain the minimum risk margin achievable, under the constraint that the number of new contracts is 1000 . It can then be considered as the maximally diversified portfolio, in the absence of geographical diversification. The maximum diversification is thus obtained by selling 1000 annuities to the UK 66 years old, whose mortality intensity process shows the 
TABLE 9

EFFECTS OF GEOGRAPHICAL DIVERSIFICATION $(r=2 \%)$.

\begin{tabular}{lcccccc}
\hline \hline Portfolio & $A V$ & $R M$ & $\Pi$ & $\% R M$ & $\sigma^{\lambda}(\Pi)$ & $D I$ \\
\hline$\Pi^{0}$ & $1.4104 \times 10^{4}$ & $1.1838 \times 10^{3}$ & $1.5288 \times 10^{4}$ & 8.39 & 0.00124 & - \\
$\Pi^{F}$ & $1.4768 \times 10^{4}$ & $1.0912 \times 10^{3}$ & $1.5586 \times 10^{4}$ & 7.39 & 0.00107 & - \\
$\Pi^{1}$ & $2.8208 \times 10^{4}$ & $2.3676 \times 10^{3}$ & $3.0576 \times 10^{4}$ & 8.39 & 0.00124 & 0 \\
$\Pi^{2}$ & $2.8872 \times 10^{4}$ & $2.2749 \times 10^{3}$ & $3.1147 \times 10^{4}$ & 7.87 & 0.00115 & 0.0746 \\
$\Pi^{3}$ & $4.3631 \times 10^{4}$ & $3.3646 \times 10^{3}$ & $4.7005 \times 10^{4}$ & 7.71 & 0.00112 & 0.0992 \\
$\Pi_{o p t}^{1}$ & $3.0187 \times 10^{4}$ & $1.9950 \times 10^{3}$ & $3.2182 \times 10^{4}$ & 6.61 & 0.00096 & 0 \\
$\Pi_{o p t}^{2}$ & $3.0790 \times 10^{4}$ & $1.8998 \times 10^{3}$ & $3.2690 \times 10^{4}$ & 6.17 & 0.00091 & 0.0121 \\
\hline \hline
\end{tabular}

minimum covariance with the other UK cohorts (see the left panel of Figure 3 ). Notice that the percentage risk margin of this portfolio is $6.61 \%$, which is lower than $7.39 \%$. Being entirely composed of UK annuitants, this portfolio has a null DI, but due to its composition, is able to reduce $\sigma^{\lambda}$ to 0.00096 .

Similarly, $\Pi_{o p t}^{2}$ is obtained allowing for geographical diversification and optimizing the composition of the foreign portfolio. The optimization is performed by looking at the covariance matrix between the two populations (see right panel of Figure 3) and choosing to concentrate the foreign expansion on the Italian 66 years old males, who have the lowest covariance with all the cohorts of the UK population. The risk margin of $\Pi_{o p t}^{2}$ is $6.17 \%$ and $\sigma^{\lambda}$ is 0.00091 . The percentage risk margin of portfolio $\Pi_{\text {opt }}^{2}$ and its $\sigma^{\lambda}$ are the lowest among the portfolios we have considered. The DI of this last portfolio is small compared to the DIs of the other portfolios involving an international expansion, being 0.0121 . It is small because the expansion is performed by concentrating the sales of policies in the foreign population in one generation only. Notice that the DI and \%RM reduction differ more when the portfolio added to the initial one is optimized across generations than when it is not. This happens because the DI - by definition, to be kept simple - does not capture the effects of putting different weights on generations with low covariance within population, while the percentage risk margin and the portfolio mortality standard deviation capture the entire dependence structure between populations and generations. Indeed, the DI provides a non-dollar measure of diversification which "averages" the contributions of different generations and penalizes any concentration in a particular one, even though the latter is justified by a strategy which aims at minimizing the risk margin reduction. This is why we presented all the three measures.

\subsection{Sensitivity analysis}

Table 10 reports the results for the different portfolios considered in Section 6.3 , under the assumption of a zero interest rate, that is $r=0 \%$. Under this lower interest rate level, the magnitude of longevity risk is more severe, as 
TABLE 10

EFFECTS OF GEOGRAPHICAL DIVERSIFICATION $(r=0 \%)$.

\begin{tabular}{lcccccc}
\hline \hline Portfolio & $A V$ & $R M$ & $\Pi$ & $\% R M$ & $\sigma^{\lambda}(\Pi)$ & $D I$ \\
\hline$\Pi^{0}$ & $1.7656 \times 10^{4}$ & $1.9408 \times 10^{3}$ & $1.9596 \times 10^{4}$ & 10.99 & 0.00124 & - \\
$\Pi^{F}$ & $1.8614 \times 10^{4}$ & $1.8109 \times 10^{3}$ & $2.0425 \times 10^{4}$ & 9.72 & 0.00107 & - \\
$\Pi^{1}$ & $3.5311 \times 10^{4}$ & $3.8815 \times 10^{3}$ & $3.9193 \times 10^{4}$ & 10.99 & 0.00124 & 0 \\
$\Pi^{2}$ & $3.6270 \times 10^{4}$ & $3.7701 \times 10^{3}$ & $4.0040 \times 10^{4}$ & 10.39 & 0.00115 & 0.0746 \\
$\Pi^{3}$ & $5.4885 \times 10^{4}$ & $5.5786 \times 10^{3}$ & $6.0464 \times 10^{4}$ & 10.16 & 0.00112 & 0.0992 \\
$\Pi_{o p t}^{1}$ & $3.8182 \times 10^{4}$ & $3.3155 \times 10^{3}$ & $4.1497 \times 10^{4}$ & 8.68 & 0.00096 & 0 \\
$\Pi_{o p t}^{2}$ & $3.9079 \times 10^{4}$ & $3.1565 \times 10^{3}$ & $4.2235 \times 10^{4}$ & 8.07 & 0.00091 & 0.0121 \\
\hline \hline
\end{tabular}

expected: the percentage Risk Margins are higher for all portfolios, increasing in the best-case scenario to $8.07 \%$, up from $6.17 \%$. However, diversification as measured by the $\% R M$ is even more valuable, because the reduction from the initial portfolio to $\Pi_{o p t}^{2}$ portfolio is almost 3 percentage points. The DI and portfolio mortality standard deviation, instead, by definition, are not affected by the change in the interest rate, because the weights appearing in (5.3) and (5.8) are expressed in nominal terms (number of annuities written on a generation) rather than in value terms (value of the annuity portfolios on the different generations, for instance).

We finally assess the impact of the parameter $\delta_{i}$ on the portfolio diversification following an expansion. In Section 6.3, we considered two countries, the UK and Italy, that belong to the same continent and share many similar features. As a consequence, also their past mortality dynamics were not so dissimilar. We expect, however, that more different countries show way lower similarity, and thus lower $\delta$ 's between cohort intensities. We perform, then, a simulation study where the parameters of the foreign population are set as in Table 2 , with the only exception of the parameters $\delta_{i}$, which we assume to be a constant $\delta$ for every generation $i$. The interest rate is set to $r=2 \%$, as in our base case. We exogenously set $\delta$ to a value that ranges from 0.1 to 0.9 . When $\delta$ is close to 0 , the dynamics of the mortality intensities of the domestic and the foreign populations are orthogonal. Thus, the international expansion targets a foreign population whose mortality dynamics is very different from the domestic one. In this case, we expect the maximum level of diversification gains from an international expansion. As $\delta$ increases, the correlation between the mortality intensities of the two populations increases as well. When $\delta$ is close to 1 , the mortality dynamics of the domestic and foreign population are perfectly correlated. In this last case, we can expect the lowest level of longevity risk diversification gains from an international expansion strategy. We compute, for each level of $\delta_{i}$, the DI, the portfolio mortality standard deviation and the percentage risk margin reduction, for the portfolios $\Pi^{2}$ and $\Pi_{o p t}^{2}$ described in Section 6.3.

As expected, the highest values of both the percentage risk margin reduction and the DI, for both portfolios, are achieved when $\delta_{i}$ is close to 0 . The 

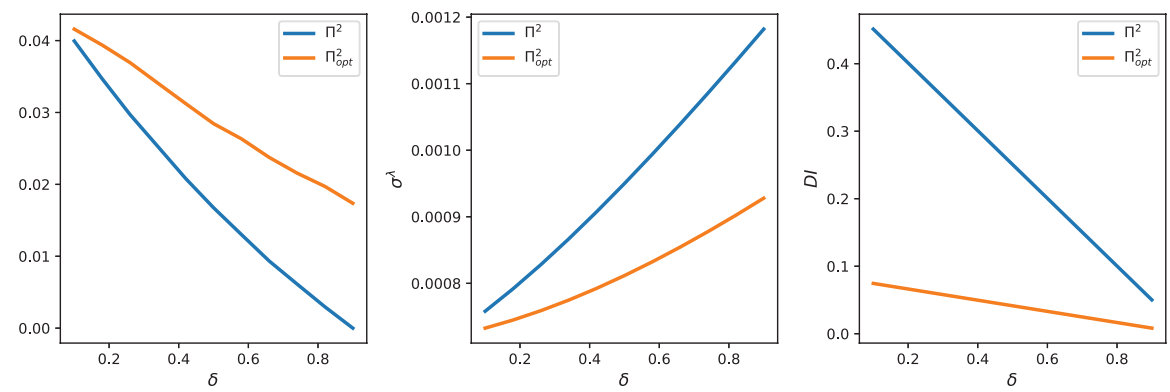

Figure 5: Left Panel: Percentage Risk Margin reduction under different exogenous $\delta_{i}=\delta$ for every $i$ assumption. Center Panel: Portfolio Mortality Standard Deviation under different exogenous $\delta_{i}=\delta$ for every $i$. Right Panel: Diversification under different exogenous $\delta_{i}=\delta$ for every $i$.

percentage risk margin reduction is $4 \%$ in this case, showing that sizable benefits from geographical diversification are possible. Such benefits, measured in terms of either the risk margin reduction or the DI, decrease as $\delta$ approaches 1 . The optimal portfolio expansion $\Pi_{\text {opt }}^{2}$ provides consistently higher risk margin reduction than $\Pi^{2}$, and the gap between the two strategies widens as $\delta$ increases (see the left panel of Figure 5). On the contrary, strategy $\Pi^{2}$ shows a higher DI with respect to $\Pi_{o p t}^{2}$, for every $\delta$.

The DI tends to 0 for both portfolios as $\delta$ goes to 1 . Instead, while the percentage risk margin reduction for $\Pi^{2}$ goes to zero when $\delta$ is 1 , portfolio expansion $\Pi_{o p t}^{2}$ offers a diversification benefit relative to the initial portfolio even in that case. This happens because the expansion is targeted in this case to a specific generation. The effects of the international expansion are analogous to those that can be obtained by targeting the domestic expansion to the generation which shows the lowest covariance with the others: $\Pi_{\text {opt }}^{2}$ reduces the percentage risk margin as much as $\Pi_{o p t}^{1}$.

Given their properties, and the evidence from this sensitivity analysis, the DI and the standard deviation of the portfolio mortality intensity can be an extremely easy-to-handle and useful tool when choosing among competing target foreign populations in an international expansion. The percentage risk margin reduction, being a percentage monetary measure of the diversification gains, is better suited, instead, to select the best strategy when different alternative foreign portfolio compositions can be targeted, once the candidate foreign population has been selected.

\section{CONCLUSiOnS}

In this paper, we discussed the benefits of geographically diversified portfolios, due to the nonperfect correlation between the dynamics of the mortality rates of different populations.

Our application, based on an annuity portfolio written on the UK and the Italian populations, shows that the effects of an international diversification are sizable. Expanding internationally decreases the volatility of the portfolio 
mortality intensity up to $26 \%$. Under a $0 \%$ interest rate assumption, we showed that an optimally designed expansion can lower the percentage risk margin, relative to the actuarial value of the portfolio, by almost 4 percentage points.

The example in the paper can be considered as conservative, since the two populations of UK and Italy present rather similar historical mortality dynamics. The diversification effect is shown to be more relevant the lower the correlation between intensities.

The diversification benefits of an international expansion may happen to be counterbalanced by the costs connected to the foreign portfolio acquisition process. These costs that are - say - the fixed costs of opening a foreign affiliate, or the fees required by the agents involved in the M \& A operation, may be substantial. As an alternative to a physical expansion, the insurer may obtain the same diversification benefit operating on the longevity derivatives market. Longevity derivatives, and longevity swaps in particular, are bespoke transactions between (re)insurers and funds or companies that agree to exchange fixed cash flows and cash flows linked to the survivorship of a particular population (see Blake et al., 2006 for instance). The buyer of the protection provided by a longevity swap transfers the longevity risk linked to a given reference population to the seller, who in turn becomes exposed to such risk. In our case, the insurer can expand internationally by receiving a fixed periodical fee and paying the realized survivorship of the foreign cohorts. Thus, the risk margin reduction benefits of a foreign expansion can be replicated by selling protection through a swap. Even in this case, however, the costs of structuring the agreement and coping with informational asymmetries (Biffis et al., 2016) can substantially reduce the diversification gains. Appendix B shows how to compare the physical and swap-based expansions, when the swap fee is fair, based on their costs.

We interpret our results as one of the possible explanations of the higher degree of internationalization of insurance companies with respect to banks after the adoption of Solvency II. Because of the synthetic possibility to diversify through longevity transfer agreements and longevity swaps, our results also explain the high number of such contracts recently signed in the marketplace and the attention dedicated to the growth of the market capacity (Blake et al., 2018).

\section{NOTES}

1. Notice that the number of parameters in the model outlined in what follows may be further reduced by linking the value of the parameters across ages using functional forms.

2. For the empirical application in Section 6, we will consider the easiest case of no dependence on the initial age $x_{i}$.

3. In principle, linear affine coefficients $a^{\prime}, b^{\prime}$, and $\sigma^{\prime}$ could be chosen.

4.

$$
\begin{aligned}
\operatorname{Var}_{0}\left(\lambda^{\Pi^{2}}(t)\right) & =\operatorname{Var}_{0}\left(\sum_{i=1}^{m} w_{i}^{d, \Pi^{2}} \lambda_{i}^{d}(t)+\sum_{i=1}^{m} w_{i}^{f, \Pi^{2}} \lambda_{i}^{f}(t)\right) \\
& =\operatorname{Var}_{0}\left(\sum_{i=1}^{m} w_{i}^{d, \Pi^{2}} \lambda_{i}^{d}(t)\right)+\operatorname{Var}_{0}\left(\sum_{i=1}^{m} w_{i}^{f, \Pi^{2}} \lambda_{i}^{f}(t)\right)+
\end{aligned}
$$




$$
\begin{aligned}
& +2 \operatorname{Cov}_{0}\left(\sum_{i=1}^{m} w_{i}^{d, \Pi^{2}} \lambda_{i}^{d}(t), \sum_{i=1}^{m} w_{i}^{f, \Pi^{2}} \lambda_{i}^{f}(t)\right) \\
& =\sum_{i=1}^{m}\left[\left(w_{i}^{d, \Pi^{2}}\right)^{2}+\left(w_{i}^{f, \Pi^{2}}\right)^{2} \delta_{i}^{2}\right] \operatorname{Var}_{0}\left(\lambda_{i}^{d}(t)\right)+ \\
& +\sum_{i=1}^{m}\left(w_{i}^{f, \Pi^{2}}\right)^{2}\left(1-\delta_{i}\right)^{2} \operatorname{Var}_{0}\left(\lambda^{\prime}(t)\right)+ \\
& +2 \sum_{i<j}\left(w_{i}^{d, \Pi^{2}} w_{j}^{d, \Pi^{2}}+w_{i}^{f, \Pi^{2}} w_{j}^{f, \Pi^{2}} \delta_{i} \delta_{j}\right) \operatorname{Cov}_{0}\left(\lambda_{i}^{d}(t), \lambda_{j}^{d}(t)\right)+ \\
& +2 \sum_{i<j} w_{i}^{f, \Pi^{2}} w_{j}^{f, \Pi^{2}}\left(1-\delta_{i}\right)\left(1-\delta_{j}\right) \operatorname{Var}_{0}\left(\lambda^{\prime}(t)\right)+ \\
& +2 \sum_{i=1}^{m} \sum_{j=1}^{m} w_{i}^{d, \Pi^{2}} w_{j}^{f, \Pi^{2}} \delta_{j} \operatorname{Cov}_{0}\left(\lambda_{i}^{d}(t), \lambda_{j}^{d}(t)\right),
\end{aligned}
$$

5. Since we are only averaging over the generations belonging to the domestic portfolio, the Similarity Index defined in Equation (5.9) should be interpreted as a synthetic measure of the similarity relative to the domestic population. If instead $m$ is defined as the number of generations in the foreign portfolio, the resulting measure should be interpreted as the similarity with respect to the foreign portfolio.

6. Indeed, by construction, the DI does not take into account the diversification benefit across different generations in the two populations.

7. The intuition behind the derivation of the $D I$ as compared to $\sigma^{\lambda}\left(\Pi^{2}\right)$ is as follows. From the definition of $\lambda^{\Pi^{2}}(t)$ and from (4.2), we observe that:

$$
\begin{aligned}
\lambda^{\Pi^{2}}(t) & =\sum_{i=1}^{m} w_{i}^{d, \Pi^{2}} \lambda_{i}^{d}(t)+\sum_{i=1}^{m} w_{i}^{f, \Pi^{2}} \lambda_{i}^{f}(t) \\
& =\sum_{i=1}^{m} w_{i}^{d, \Pi^{2}} \lambda_{i}^{d}(t)+\sum_{i=1}^{m} w_{i}^{f, \Pi^{2}} \delta_{i} \lambda_{i}^{d}(t)+\sum_{i=1}^{m} w_{i}^{f, \Pi^{2}}\left(1-\delta_{i}\right) \lambda^{\prime}(t) .
\end{aligned}
$$

The last term in (7.9) can be interpreted as the source of the diversification benefit, and each coefficient of the summation $w_{i}^{f, \Pi^{2}}\left(1-\delta_{i}\right)$ can be interpreted as the diversification contribution of each foreign generation $i$. Hence, the $D I$ can be seen as the average diversification contribution of each generation in the foreign portfolio.

As shown by diagram (A.17) in Appendix A for the dependence structure between the foreign and domestic generations, we can say that $\sigma^{\lambda}\left(\Pi^{2}\right)$ captures both the horizontal and the vertical dependence, while $D I$ only focuses on the first one.

8. These correspond to the last 20 observations available to date for the Italian males. However, since the UK data set is updated until 31/12/2013, we have excluded the last available observation for the UK cohorts.

9. In an unreported simulated robustness analysis, we find a Mean Absolute Percentage Error of the Gaussian Mapping estimates of about $9 \%$ consistently across all age pairs.

10. Source: Human Mortality Database.

11. Inserting the exact composition of the UK population is a trivial extension.

\section{REFERENCES}

Biffis, E., Blake, D., Pitotti, L. and Sun, A. (2016) The cost of counterparty risk and collateralization in longevity swaps. Journal of Risk and Insurance, 83(2), 387-419.

Blake, D., Cairns, A., Dowd, K. and MacMinn, R. (2006) Longevity bonds: Financial engineering, valuation, and hedging. Journal of Risk and Insurance, 73(4), 647-672. 
Blake, D., Cairns, A.J.G., Dowd, K. and Kessler, A.R. (2018) Still living with mortality: The longevity risk transfer market after one decade. British Actuarial Journal, 24, 1-80.

BRigo, D. and Alfonsi, A. (2005) Credit default swap calibration and derivatives pricing with the ssrd stochastic intensity model. Finance and Stochastics, 9(1), 29-42.

Brigo, D. and Mercurio, F. (2001) Interest Rate Models: Theory and Practice. Springer Finance. Berlin, Heidelberg, Paris: Springer.

Cox, J.C., Ingersoll JR, J.E. and Ross, S.A. (1985) An intertemporal general equilibrium model of asset prices. Econometrica: Journal of the Econometric Society, 53(2), 363-384.

Cummins, J.D., Tennyson, S. and Weiss, M.A. (1999) Consolidation and efficiency in the us life insurance industry. Journal of Banking \& Finance, 23(2), 325-357.

Cummins, J.D. and XIE, X. (2008) Mergers and acquisitions in the us property-liability insurance industry: Productivity and efficiency effects. Journal of Banking \& Finance, 32(1), 30-55.

Dahl, M., Melchior, M. and Møller, T. (2008) On systematic mortality risk and riskminimization with survivor swaps. Scandinavian Actuarial Journal, 2008(2-3), 114-146.

De Rosa, C., Luciano, E. and ReGis, L. (2017) Basis risk in static versus dynamic longevityrisk hedging. Scandinavian Actuarial Journal, 2017(4), 343-365.

De Rosa, C., Luciano, E. and Regis, L. (2018) International longevity risk pooling. In Mathematical and Statistical Methods for Actuarial Sciences and Finance, pp. 317-321. Springer.

Enchev, V., Kleinow, T. and CAirns, A.J. (2017) Multi-population mortality models: Fitting, forecasting and comparisons. Scandinavian Actuarial Journal, 2017(4), 319-342.

Haberman, S., Kaishev, V., Millosovich, P., Villegas, A., Baxter, S., Gaches, A., Gunnlaugsson, S. and Sison, M. (2014) Longevity basis risk: A methodology for assessing basis risk. Institute and Faculty of Actuaries (IFoA) Sessional Research Paper.

JeVtić, P. and REGis, L. (2019) A continuous-time stochastic model for the mortality surface of multiple populations. Insurance: Mathematics and Economics, 88, 181-195.

LEE, R.D. and CARTER, L.R. (1992) Modeling and forecasting us mortality. Journal of the American Statistical Association, 87(419), 659-671.

LI, N. and Lee, R. (2005) Coherent mortality forecasts for a group of populations: An extension of the lee-carter method. Demography, 42(3), 575-594.

Luciano, E., Regis, L. and Vigna, E. (2012) Delta-gamma hedging of mortality and interest rate risk. Insurance: Mathematics and Economics, 50(3), 402-412.

Luciano, E., Spreeuw, J. and Vigna, E. (2008) Modelling stochastic mortality for dependent lives. Insurance: Mathematics and Economics, 43(2), 234-244.

MA, Y.-L. and POPE, N. (2003) Determinants of international insurers' participation in foreign non-life markets. Journal of Risk and Insurance, 70(2), 235-248.

Milevsky, M.A. and Promislow, S.D. (2001) Mortality derivatives and the option to annuitise. Insurance: Mathematics and Economics, 29(3), 299-318.

SCHOENMAKER, D. and SASS, J. (2016) Cross-border insurance in europe: Challenges for supervision. The Geneva Papers on Risk and Insurance-Issues and Practice, 41(3), 351-377.

Sher RIS, M., XU, Y. and Ziveyi, J. (2020) Cohort and value-based multi-country longevity risk management. Scandinavian Actuarial Journal, 2020(7), 650-676.

YANG, S.S. and WANG, C.-W. (2013) Pricing and securitization of multi-country longevity risk with mortality dependence. Insurance: Mathematics and Economics, 52(2), 157-169.

Clemente De Rosa

Scuola Normale Superiore

Piazza dei Cavalieri 7

56126 Pisa, Italy

E-Mail: clemente.derosa@sns.it 


\section{ELISA LUCIANO}

ESOMAS Department

University of Torino, Collegio Carlo Alberto and Institut Louis Bachelier

Corso Unione Sovietica 218/bis

10134, Torino, Italy

E-Mail: elisa.luciano@unito.it

LUCA REGIS (Corresponding author)

ESOMAS Department

University of Torino and Collegio Carlo Alberto

Corso Unione Sovietica 218/bis

10134, Torino, Italy

E-Mail: luca.regis@unito.it

\section{APPENDIX A. VARIANCE COVARIANCE STRUCTURE OF THE MORTALITY INTENSITIES}

The time-t variance of the intensity of a generation $i$ belonging to the domestic population, $\lambda_{i}^{d}$, conditional on the information at time 0 is available in closed form and is equal to

$$
\operatorname{Var}_{0}\left(\lambda_{i}^{d}(t)\right)=\frac{a_{i} \sigma_{i}^{2}}{2 b_{i}^{2}}\left(e^{b_{i} t}-1\right)^{2}+\frac{\sigma_{i}^{2}}{b_{i}} e^{b_{i} t}\left(e^{b_{i} t}-1\right) \lambda_{i}^{d}(0) .
$$

Similarly, the conditional variance of $\lambda_{i}^{f}(t)$ is

$$
\operatorname{Var}_{0}\left(\lambda_{i}^{f}(t)\right)=\delta_{i}^{2} \operatorname{Var}_{0}\left(\lambda_{i}^{d}(t)\right)+\left(1-\delta_{i}\right)^{2} \operatorname{Var}_{0}\left(\lambda^{\prime}(t)\right)
$$

where

$$
\operatorname{Var}_{0}\left(\lambda^{\prime}(t)\right)=\frac{a^{\prime}\left(\sigma^{\prime}\right)^{2}}{2\left(b^{\prime}\right)^{2}}\left(e^{b^{\prime} t}-1\right)^{2}+\frac{\left(\sigma^{\prime}\right)^{2}}{b^{\prime}} e^{b^{\prime} t}\left(e^{b^{\prime} t}-1\right) \lambda^{\prime}(0) .
$$

Since the mortality of the domestic generations follow a square-root process, there is no closed-form expression for the covariance between the intensities of the two generations $i$ and $j$. However, we can obtain a closed-form approximation using the Gaussian Mapping (Brigo and Alfonsi, 2005) technique, as follows. For each CIR process, we consider a Vasicek process driven by the same Brownian Motion $W_{i}(t)$, having the same drift and the same initial point of the CIR process:

$$
d \lambda_{i}^{V}(t)=\left(a_{i}+b_{i} \lambda_{i}^{V}(t)\right) d t+\sigma_{i}^{V} d W_{i}(t), \quad \lambda_{i}^{V}(0)=\lambda_{i}^{d}(0) .
$$

The instantaneous volatility coefficient $\sigma_{i}^{V}$ of (A.4) is then determined by making the two processes as close as possible, in the sense that they return the same survival probability:

$$
S_{i}^{d}(t, T)=S_{i}^{V}\left(t, T ; \sigma_{i}^{V}\right)
$$


The technique relies on the application of Itô's Lemma to the solution to the SDE (A.4), which is given by:

$$
\lambda_{i}^{V}(t)=\lambda_{i}^{V}(0) e^{b_{i} t}+\frac{a_{i}}{b_{i}}\left(1-e^{b_{i} t}\right)+\sigma_{i}^{V} \int_{0}^{t} e^{b_{i}(t-s)} d W_{i}(s) .
$$

Therefore, we have that:

$$
\begin{aligned}
\mathbb{E}_{0}\left[\lambda_{i}^{V}(t)\right] & =\lambda_{i}^{V}(0) e^{b_{i} t}+\frac{a_{i}}{b_{i}}\left(1-e^{b_{i} t}\right) \\
\operatorname{Var}_{0}\left[\lambda_{i}^{V}(t)\right] & =\frac{\left(\sigma_{i}^{V}\right)^{2}}{2 b_{i}} \sqrt{e^{2 b_{i} t}-1} .
\end{aligned}
$$

Since $\lambda_{i}^{V}(t)-\mathbb{E}_{0}\left[\lambda_{i}^{V}(t)\right]=\sigma_{i}^{V} \int_{0}^{t} e^{b_{i}(t-s)} d W_{i}(s)$, the covariance between $\lambda_{i}^{V}(t)$ and $\lambda_{j}^{V}(t)$ is:

$$
\begin{aligned}
\operatorname{Cov}_{0}\left(\lambda_{i}^{V}(t), \lambda_{j}^{V}(t)\right) & =\mathbb{E}_{0}\left[\sigma_{i}^{V} \sigma_{j}^{V}\left(\int_{0}^{t} e^{b_{i}(t-s)} d W_{i}(s)\right)\left(\int_{0}^{t} e^{b_{j}(t-s)} d W_{j}(s)\right)\right] \\
& =\mathbb{E}_{0}\left[\sigma_{i}^{V} \sigma_{j}^{V} \rho_{i j} \int_{0}^{t} e^{\left(b_{i}+b_{j}\right)(t-s)} d s\right] \\
& =\sigma_{i}^{V} \sigma_{j}^{V} \rho_{i j} \int_{0}^{t} e^{\left(b_{i}+b_{j}\right)(t-s)} d s \\
& =\frac{\sigma_{i}^{V} \sigma_{j}^{V} \rho_{i j}}{b_{i}+b_{j}}\left(e^{\left(b_{i}+b_{j}\right) t}-1\right) .
\end{aligned}
$$

Finally, we have:

$$
\begin{aligned}
\operatorname{Corr}_{0}\left(\lambda_{i}^{V}(t), \lambda_{j}^{V}(t)\right) & =\frac{\operatorname{Cov}_{0}\left(\lambda_{i}^{V}(t), \lambda_{j}^{V}(t)\right)}{\sqrt{\operatorname{Var}_{0}\left[\lambda_{i}^{V}(t)\right] \operatorname{Var}_{0}\left[\lambda_{j}^{V}(t)\right]}} \\
& =\frac{2 \rho_{i j}}{b_{i}+b_{j}} \cdot \frac{e^{\left(b_{i}+b_{j}\right) t}-1}{\sqrt{\frac{\left(e^{2 b_{i} t}-1\right)\left(e^{2 b_{j} t}-1\right)}{b_{i} b_{j}}}} .
\end{aligned}
$$

According to such technique, we have that

$$
\operatorname{Cov}_{0}\left(\lambda_{i}^{d}(t), \lambda_{j}^{d}(t)\right)=\frac{\sigma_{i}^{V} \sigma_{j}^{V} \rho_{i j}}{b_{i}+b_{j}}\left(e^{\left(b_{i}+b_{j}\right) t}-1\right),
$$

where $\sigma_{i}^{V}$ and $\sigma_{j}^{V}$ are the instantaneous volatilities resulting from the mapping of $\lambda_{i}^{d}$ and $\lambda_{j}^{d}$ into Gaussian processes.

From (4.1) and (4.2) we have that the covariance between the same generation $i$ belonging to the domestic and foreign population can be written as:

$$
\operatorname{Cov}_{0}\left(\lambda_{i}^{d}(t), \lambda_{i}^{f}(t)\right)=\delta_{i} \operatorname{Var}_{0}\left(\lambda_{i}^{d}(t)\right) .
$$

Considering, instead, two different generations $i$ and $j$ belonging to the foreign population, we have that

$$
\operatorname{Cov}_{0}\left(\lambda_{i}^{f}(t), \lambda_{j}^{f}(t)\right)=\delta_{i} \delta_{j} \operatorname{Cov}_{0}\left(\lambda_{i}^{d}(t), \lambda_{j}^{d}(t)\right)+\left(1-\delta_{i}\right)\left(1-\delta_{j}\right) \operatorname{Var}_{0}\left(\lambda^{\prime}(t)\right) .
$$


Finally, the covariance between the mortality intensity of generation $i$ belonging to the foreign population and generation $j$ belonging to the domestic is given by:

$$
\operatorname{Cov}_{0}\left(\lambda_{i}^{f}(t), \lambda_{j}^{d}(t)\right)=\delta_{i} \operatorname{Cov}_{0}\left(\lambda_{i}^{d}(t), \lambda_{j}^{d}(t)\right) .
$$

Thanks to the Gaussian Mapping technique we can also compute the conditional correlation between two generations belonging to two different populations. Considering $0 \leq u \leq t$, the conditional correlation between $\lambda_{x_{i}}^{d}(t)$ and $\lambda_{x_{j}}^{f}(t)$ is given by:

$$
\operatorname{Corr}_{u}\left[\lambda_{x_{i}}^{d}(t), \lambda_{x_{j}}^{f}(t)\right]=\delta_{j} \frac{\operatorname{Cov}_{u}\left(\lambda_{x_{i}}^{d}(t), \lambda_{x_{j}}^{d}(t)\right)}{\sqrt{\operatorname{Var}_{u}\left(\lambda_{x_{i}}^{d}(t)\right) \cdot \operatorname{Var}_{u}\left(\lambda_{x_{j}}^{f}(t)\right)}},
$$

where $\operatorname{Cov}_{u}\left(\lambda_{x_{i}}^{d}(t), \lambda_{x_{j}}^{d}(t)\right)$ is computed using the Gaussian mapping technique, and

$$
\operatorname{Var}_{u}\left(\lambda_{x_{j}}^{f}(t)\right)=\delta_{j}^{2} \operatorname{Var}_{u}\left(\lambda_{x_{j}}^{d}(t)\right)+\left(1-\delta_{j}\right)^{2} \operatorname{Var}_{u}\left(\lambda^{\prime}\left(t ; x_{j}\right)\right)
$$

From (A.14), it is interesting to notice that the covariance between $\lambda_{i}^{f}$ and $\lambda_{j}^{d}$ depends both on $\delta_{i}$, which measures the dependence between the same generation $i$ across the two populations, and on $\operatorname{Cov}_{0}\left(\lambda_{i}^{d}, \lambda_{j}^{d}\right)$, which instead measures the dependence between the generations $i$ and $j$ within the domestic population.

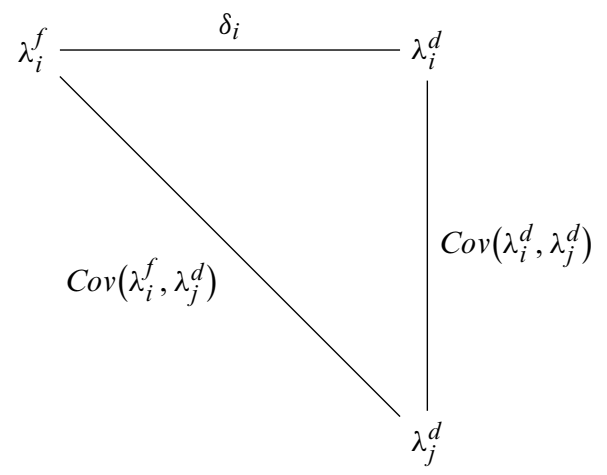

(A.17)

Diagram (A.17) visualizes that, when computing the covariance $\lambda_{i}^{f}$ and $\lambda_{j}^{d}$, we are able to disentangle the effect of the two types of dependence: the within-population and the crosspopulation ones. The importance of (A.17) can be explained with a simple example. Suppose there are two portfolios belonging to two populations $f_{1}$ and $f_{2}$ that are competing targets of a foreign expansion, and suppose that each portfolio is composed of annuities sold only to one generation $k$. The objective of the expansion is to find a foreign portfolio that minimizes $\operatorname{Cov}\left(\lambda_{k}^{f_{*}}, \lambda_{j}^{d}\right)$, to obtain the maximum level of longevity risk diversification. Since we cannot change the covariance structure of the domestic population, the solution to the problem is to find the portfolio $\Pi^{f_{*}}$ such that

$$
f_{*}=\arg \min _{x \in\left\{f_{1}, f_{2}\right\}}\left(\delta_{k}^{x}\right) .
$$


Then, it is sufficient to compare the $\delta$ 's of the two competing foreign populations. For instance, if $\delta_{k}^{f_{1}}<\delta_{k}^{f_{2}}$, then the optimal foreign expansion target portfolio is $\Pi^{f_{1}}$.

\section{APPENDIX B. PHYSICAL VERSUS SYNTHETIC EXPANSION}

A foreign portfolio expansion like the one we considered with portfolio $\Pi_{o p t}^{2}$ is in reality very difficult to achieve, because it is unlikely that an insurer can target the sale of annuity contracts only to a specific cohort. Even if the expansion is feasible, it is likely to entail some $\operatorname{cost} C_{0}>0$. Therefore, the real value of the liabilities of the insurer following the optimal foreign portfolio expansion can be rewritten as:

$$
\bar{\Pi}_{o p t}^{2}=\Pi_{o p t}^{2}+C_{0}
$$

On the other hand, the same level of geographical diversification could be synthetically obtained through a longevity swap. Consider our UK life insurer with portfolio $\Pi^{0}$, and assume that she sells a longevity swap written on 1000 individuals aged 66 belonging to the Italian population. Being the seller of the swap, the UK insurer will receive every year, until the maturity of the contract, a fixed amount equal to $K$ and will pay a stochastic amount given by the realized survival rate of the Italian 66 years old males. Let the maturity of the swap be $T=\omega$ and assume independence between mortality and interest rate risk. From the point of view of the seller, the value at time $t$ of the longevity swap is:

$$
\begin{aligned}
L(t, T) & =1000 \sum_{\mathcal{T}=t+1}^{T-t}\left[K-S_{66}(t, \mathcal{T})\right] D(t, \mathcal{T})= \\
& =1000 \sum_{\mathcal{T}=t+1}^{T-t} \mathbb{E}_{t}\left[K-\exp \left(-\int_{t}^{\mathcal{T}} \lambda_{66}(s) d s\right)\right] \mathbb{E}_{t}\left[\exp \left(-\int_{t}^{\mathcal{T}} r(u) d u\right)\right]
\end{aligned}
$$

where $K$ is the swap rate, $S_{66}(t, \mathcal{T})$ is the $(t, \mathcal{T})$-Survival probability for a 66 years old Italian male and $D(t, \mathcal{T})$ is the discount factor. If the swap is fairly priced, the swap rate is chosen in such a way that the value of the contract is zero at inception, that is:

$$
K=\frac{\sum_{\mathcal{T}=1}^{T-t} \mathbb{E}\left[\exp \left(-\int_{0}^{\mathcal{T}} \lambda_{66}(s) d s\right)\right] \mathbb{E}\left[\exp \left(-\int_{0}^{\mathcal{T}} r(u) d u\right)\right]}{\sum_{\mathcal{T}=1}^{T-t} \mathbb{E}\left[\exp \left(-\int_{0}^{\mathcal{T}} r(u) d u\right)\right]} .
$$

In our calibration, assuming a constant interest rate of $2 \%$, we have that $K=0.7295$.

The actuarial value of $\tilde{\Pi}_{o p t}^{2}$ is then

$$
A V_{\tilde{\Pi}_{o p t}^{2}}(t)=A V_{\Pi_{o p t}^{2}}(t)
$$


The risk margin of $\tilde{\Pi}_{o p t}^{2}$ is:

$$
\begin{aligned}
R M_{\tilde{\Pi}_{o p t}^{2}}(t) & =D(t, t+T) \cdot V a R_{\alpha}\left(A V_{\tilde{\Pi}_{o p t}^{2}}(t+T)-\mathbb{E}_{t}\left[A V_{\tilde{\Pi}_{o p t}^{2}}(t+T)\right]\right) \\
& =D(t, t+T) \cdot V a R_{\alpha}\left(A V_{\Pi_{o p t}^{2}}(t+T)-\mathbb{E}_{t}\left[A V_{\Pi_{o p t}^{2}}(t+T)\right]\right) \\
& =R M_{\Pi_{o p t}^{2}}(t) .
\end{aligned}
$$

So, if fairly priced at inception, the longevity swap allows the insurer to achieve the same actuarial value and the same risk margin of a physical sale of annuity contracts to the Italian males. The sales of the longevity swap may entail some initial cost $C_{0}^{\prime}$ given, for instance, by the required due diligence actions. Hence, the value of the liability portfolio $\tilde{\Pi}_{o p t}^{2}$ is given by:

$$
\tilde{\Pi}_{o p t}^{2}=\Pi_{o p t}^{2}+C_{0}^{\prime} .
$$

As long as $C_{0}^{\prime}<C_{0}$, the UK insurer will find in the synthetic expansion trough the longevity swap a more attractive solution. 